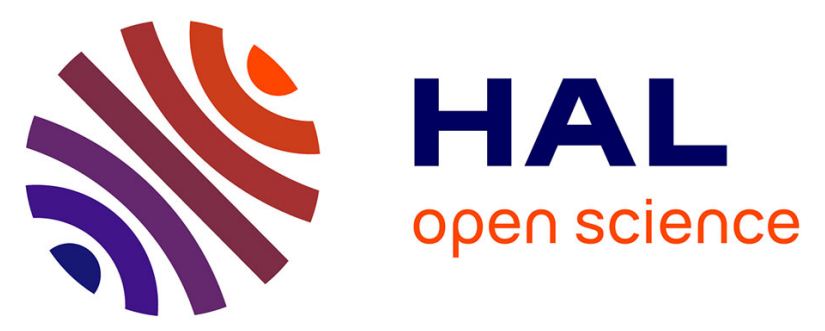

\title{
Comparison of dissolved inorganic and organic carbon yields and fluxes in the watersheds of tropical volcanic islands, examples from Guadeloupe (French West Indies)
}

E. Lloret, C. Dessert, Jérôme Gaillardet, Patrick Albéric, O. Crispi, C. Chatudeau, M.F. Benedetti

\section{To cite this version:}

E. Lloret, C. Dessert, Jérôme Gaillardet, Patrick Albéric, O. Crispi, et al.. Comparison of dissolved inorganic and organic carbon yields and fluxes in the watersheds of tropical volcanic islands, examples from Guadeloupe (French West Indies). Chemical Geology, 2011, 280 (1-2), pp.65-78. 10.1016/j.chemgeo.2010.10.016 . insu-00536476

HAL Id: insu-00536476

https://hal-insu.archives-ouvertes.fr/insu-00536476

Submitted on 22 Nov 2012

HAL is a multi-disciplinary open access archive for the deposit and dissemination of scientific research documents, whether they are published or not. The documents may come from teaching and research institutions in France or abroad, or from public or private research centers.
L'archive ouverte pluridisciplinaire HAL, est destinée au dépôt et à la diffusion de documents scientifiques de niveau recherche, publiés ou non, émanant des établissements d'enseignement et de recherche français ou étrangers, des laboratoires publics ou privés. 


\title{
Comparison of dissolved inorganic and organic carbon yields and fluxes in the watersheds of tropical volcanic islands, examples from Guadeloupe (French West Indies)
}

\author{
E. Lloret $^{\mathrm{a},}$ C. Dessert ${ }^{\mathrm{b}}{ }^{\mathrm{c}}$ J. Gaillardet ${ }^{\mathrm{b}}$ P. Albéric $^{\mathrm{d}}$ O. Crispi $^{\mathrm{c}}$ C. Chaduteau ${ }^{\mathrm{e}}$ M.F. Benedetti ${ }^{\mathrm{a}}$ \\ ${ }^{a}$ Equipe Géochimie des Eaux, Université Paris-Diderot, IPGP, UMR CNRS 7154, 75205 \\ Paris, France \\ ${ }^{\mathrm{b}}$ Equipe de Géochimie et Cosmochimie, IPGP, Université Paris-Diderot, Sorbonne Paris \\ Cité, CNRS (UMR 7154), 75252 Paris, France \\ ${ }^{c}$ Observatoire Volcanologique et Sismologique de Guadeloupe (OVSG) IPGP, UMR \\ CNRS 7154, Le Houëlmont, 97113 Gourbeyre, Guadeloupe (FWI), France \\ ${ }^{\mathrm{d}}$ Institut des Sciences de la Terre d'Orléans (ISTO), Université d'Orléans, CNRS (UMR \\ 6113), Campus Géosciences, 45071 Orléans, France
}

${ }^{\mathrm{e}}$ Equipe de Physico-chimie des fluides géologiques, IPGP, Université Paris-Diderot, Sorbonne Paris Cité, CNRS (UMR 7154), 75252 Paris, France

\begin{abstract}
Organic matter is an important factor that cannot be neglected when considering global carbon cycle. New data including organic matter geochemistry at the small watershed scale are needed to elaborate more constrained carbon cycle and climatic models. The objectives are to estimate the DOC and DIC yields exported from small tropical watersheds and to give strong constraints on the carbon hydrodynamic of these systems. To answer these questions, we have studied the geochemistry of eleven small watersheds around Basse-Terre volcanic Island in the French West Indies during different hydrological regimes from 2006 to 2008 (i.e. low water level versus floods). We propose a complete set of carbon measurements, including DOC and DIC concentrations, $\delta^{13} \mathrm{C}$ data, and less commonly, some spectroscopic indicators of the nature of organic matter. The DOC/DIC ratio varies between 0.07 and 0.30 in low water level and between 0.25 and 1.97 during floods, indicating that organic matter is mainly exported during flood events. On the light of the isotopic composition of DOC, ranging from -32.8 to $-26.2 \%$ during low water level and from -30.1 to $-27.2 \%$ during floods, we demonstrate that export of organic carbon is mainly controlled by perennial saprolite groundwaters, except for flood events during which rivers are also strongly influenced by soil erosion. The mean annual yields ranged from 2.5 to $5.7 \mathrm{t} \mathrm{km}^{-2}$ year $^{-1}$ for the DOC and from 4.8 to $19.6 \mathrm{t} \mathrm{km}^{-2}$ year $^{-1}$ for the DIC and exhibit a non-linear relationship with slopes of watersheds. The flash floods explain around $60 \%$ of the annual DOC flux and between 25 and $45 \%$ of the DIC flux, highlighting the important role of these extreme meteorological events on global carbon export in small tropical volcanic islands. From a carbon mass balance point of view the exports of dissolved carbon from small volcanic islands are important and should be included in global organic carbon budgets.
\end{abstract}

\section{Research Highlights}

Source of DOC is mainly controlled by saprolite groundwaters, except for flood events. 
The mean annual yields exhibit a non-linear relationship with slopes of watersheds.

The flash floods account for $60 \%$ of the annual DOC flux and $40 \%$ of the DIC flux.

The DOC input of small volcanic islands is similar to the Amazon flux to the ocean.

\title{
Keywords
}

\author{
Organic matter; Carbon fluxes; DIC; DOC; $\delta^{13} \mathrm{C}$; Tropical rivers; Watersheds
}

\section{Introduction}

At the Earth's surface, a suite of chemical, physical and biological processes are closely combined to transform bedrock into soil. This complex engine regulates the rates of soil production through chemical weathering and organic productivity, and the rates of soil destruction through physical erosion at all temporal and spatial scales. Then it exerts a major control on the transport of the dissolved and particulate products of mechanical erosion and chemical weathering from continents to the oceans and influences the biogeochemical cycles of many elements, including carbon. As shown in pioneering works (Berner et al., 1983), chemical weathering of silicate rocks converts atmospheric $\mathrm{CO}_{2}$ into bicarbonates within the stream. This process then strongly affects carbon cycle and many studies have focused on river geochemistry to quantify and characterize silicate weathering fluxes and associated inorganic carbon fluxes (e.g. [Stallard and Edmond, 1983], [Dupré et al., 1996], [Drever, 1997], [Gaillardet et al., 1999a], [Dessert et al., 2001] and [Viers et al., 2007]).

These previous studies mainly focus on the inorganic part of the carbon cycling and its role on weathering and its impact on climate change. A part of this cycle, however also concerns the organic carbon pool and cannot be neglected when considering the global carbon cycle at geological time scale. Indeed, total organic carbon flux (dissolved and particulate) from continents to the oceans represents globally $40 \%$ of the total carbon flux (i.e. 0.4 to $0.9 \mathrm{Gt} \mathrm{yr}^{-1}$; [Hedges et al., 1997], [Schlunz and Schneider, 2000] and [Aitkenhead-Peterson et al., 2003]). Moreover, soil organic matter with 1400 to $1500 \mathrm{Gt}$ of carbon is one of the major pools of carbon at the Earth surface ( [Schlesinger, 1977] and [Gregory et al., 1999]). The soil erosion is therefore the major input of organic carbon in aquatic ecosystems. During its transport to the oceans, the terrestrial organic carbon can be mineralized, or deposited and stored in aquatic ecosystems under low discharge (i.e.: alluvial plains, mangroves; Lal, 2004). This terrestrial organic matter is then mineralized or preserved in continental margins, and/or transported offshore (Hedges et al., 1997). Actually, an average of $20 \%$ of the flux of terrestrial dissolved organic carbon $\left(0.25 \mathrm{Gt} \mathrm{yr}^{-1}\right)$ is removed in estuaries with a wide range of 4-60\% that depends of the discharged organic matter ( [Hedges et al., 1997], [Schlunz and Schneider, 2000] and [Burdige, 2005]). Previous works on the fate of dissolved organic matter have shown considerable mixing gradient and high short-term variability. In addition, slow photolysis and microbial degradation are major processes that will also contribute to dissolved organic matter removal in coastal and surface ocean waters ( [Hedges et al., 1997] and [Benner, 2004]). Therefore, it is of major importance to understand the dissolved organic carbon dynamic within and along the river system (sources, fluxes and sinks) to better assess the global carbon cycle.

Currently, studies concerning the organic carbon cycle and its impact on global carbon cycle are often focused on soil erosion ( [Lal, 2003], [Lal, 2005] and [Mills and Cowling, 2010]) 
and deposit of terrestrial particulate organic carbon in marine sediments ( [France-Lanord and Derry, 1994], [Goñi et al., 1998], [Galy et al., 2008] and [Hilton et al., 2010]). Recent studies ( [Waterloo et al., 2006], [Dawson et al., 2008], [Goldsmith et al., 2008] and [Hilton et al., 2008]) showed that the export of particulate organic carbon depends on the runoff and rainfalls. Indeed, during rainfalls, the top of soil profile enriched in organic carbon is lixiviated, and carbon is transferred into the rivers (Batjes, 1996).

Regarding rivers, studies are generally focused on large river systems like the Mississippi ( [Bianchi et al., 2007] and [Duan et al., 2007]), the Ganga-Brahmaputra (Galy et al., 2008), tributaries of the Amazon river ( [Moreira-Turcq et al., 2003], [Johnson et al., 2006] and [Aufdenkampe et al., 2007]), large Arctic rivers (Yenisey, Ob, Lena; [Ludwig et al., 1996a], [Dittmar and Kattner, 2003], [Gebhardt et al., 2004] and [Raymond et al., 2007]), ... which integrate differences in lithologies, vegetations, soils and climates. However, it was underlined that the small mountainous rivers could also be very important in transporting organic sediments to oceans, fluxes being inversely proportional to the watershed area ( [Milliman and Meade, 1983], [Walling, 1983], [Degens and Ittekkot, 1985] and [Milliman and Syvitski, 1992]).

Moreover, it is difficult for studies about large rivers to constrain the different processes (natural vs land use; Amiotte-Suchet et al., 2007) influencing the organic carbon cycle as well as the weathering engine. For instance, the dissolved organic carbon can influence the bedrock weathering, since organic acids produced by microbially communities may accelerate mineral dissolution ( [Bennett et al., 1988], [Drever, 1994], [Pittman and Lewan, 1994], [Drever and Stillings, 1997], [Viers et al., 1997], [Oliva et al., 1999], [Welch and Ullman, 2000] and [Millot et al., 2003]). The major sources of organic carbon in rivers are: soil organic matter, generated by the partial or total decomposition of living organisms (plants, animals and microorganisms); primary production in the river; the decomposition of living organism in the river (algae and aquatic animals); and groundwater (Battin et al., 2008). The organic carbon quality and concentrations in rivers depend therefore on different parameters (i.e. temperature, soil types, vegetation types and bedrock) controlling the distribution of these different sources. The recent study of Raymond et al. (2007) showed that arctic watersheds show a marked increase in dissolved organic carbon concentration with flow.

Wet tropical regions are ideal targets to study the organic carbon cycle. They have optimal climatic conditions for the volcanic rock weathering, including high temperatures and high runoff, promoting the transformation of rocks into soils ( [Stallard, 1988], [Benedetti et al., 1994], [Louvat and Allègre, 1997], [Dessert et al., 2001], [Dessert et al., 2003], [Rad et al., 2006] and [Goldsmith et al., 2010]). Wet tropical regions also host the largest stock of organic carbon since $26 \%$ of the total global carbon in soils (depth from 0 to $200 \mathrm{~cm}$ ) is found in these regions (Batjes, 1996). Moreover, the export of inorganic and organic carbon is estimated to be the highest in wet tropical regions ( [Ludwig et al., 1996a], [Ludwig et al., 1996b], [Gaillardet et al., 1999b] and [Dessert et al., 2003]). Different authors have demonstrated the importance of oceanic "high standing" islands (HSIs) for sediments and total organic carbon export, for example in the southwest Pacific (i.e. 33\% of total sediment entering the world's oceans annually, Milliman and Syvitski, 1992) and Oceania's small mountainous rivers (i. e. $21-38 \%$ of the total oceanic organic carbon inputs, Schlunz and Schneider, 2000). Therefore, these small mountainous rivers and HSIs are ideal settings to study geochemical fluxes. Moreover, previous studies on weathering rates of HSIs have shown some of the highest observed rates of chemical weathering and $\mathrm{CO}_{2}$ consumption ( [Jacobson et al., 2003] and [Lyons et al., 2005]). The Guadeloupe Island (French West Indies) is an ideal 
location to study geochemical fluxes and the organic carbon geochemistry because its monolithologic volcanic composition helps to constrain the influence of other factors such as climate, soil composition, and age of the bedrock. In addition, its location in a wet tropical area implies that rates of chemical weathering and mechanical denudation are high ( [Louvat, 1997], [Louvat and Allègre, 1997], [Dessert et al., 2001], [Dessert et al., 2003], [Rad et al., 2007] and [Goldsmith et al., 2010]). Previous works of [Rad et al., 2006] and [Goldsmith et al., 2010] were focused on stream geochemistry, weathering and $\mathrm{CO}_{2}$ consumption in rivers of andesitic terrains without addressing the effect of the hydrological variability. Moreover, due to their small size, and steep slopes, the Guadeloupean rivers present a characteristic flash flood hydrological regime ( [Ibiza, 1984-1985] and [Pagney, 1994]). The dynamic of carbon export could be different from one hydrological regime to another (i.e. low water level vs flood level). Our overarching goal is to obtain new data that will help to elaborate more constrained coupled modeling of biological and chemical weathering processes at the continental scale such as B-WITCH (Roelandt et al., 2010), that mostly focus on inorganic carbon. The collected data will also help to constrain the residence time of carbon in tropical soils and to understand the fate of terrestrial DOC in the ocean.

The aims of this paper are, with major elements, dissolved inorganic (DIC) and dissolved organic (DOC) carbon concentrations, (1) to calculate the DOC and DIC yields and fluxes in Guadeloupean rivers, (2) to characterize and identify the different sources of the organic carbon exported under different hydrological regimes (i.e. low water level vs flood level) and, (3) to evidence spatial and/or temporal variations. The comparison between two hydrological regimes is therefore needed for a better assessment of weathering processes and carbon fluxes and we emphasize the importance of DOC fluxes in the carbon mass balance in small mountainous tropical rivers.

\section{General settings of Basse-Terre Island}

The Basse-Terre Island is the volcanic part of the Guadeloupe archipelago, French West Indies, with an area of $950 \mathrm{~km}^{2}$ (Fig. 1). The Lesser Antilles arc volcanism is generated by the subduction of the Atlantic seafloor beneath the Caribbean plate at a rate of about $2 \mathrm{~cm} \mathrm{yr}^{-1}$ (e.g. [Jordan, 1975], [Hawkesworth and Powell, 1980], [Wadge, 1984] and [DeMets et al., 2000]). The major part of the volcanic material of the Island is andesitic. The geology of the Basse-Terre was described by [De Reynal de Saint-Michel, 1966], [Westercamp and Tazieff, 1980] and [Boudon et al., 1988], and Westercamp (1988) and the ages of geological formations were recently reevaluated by Samper et al. (2007). The North of the island is characterized by a Pliocene volcanism (2.7-1.5 Myrs), with the series following: volcanic formations (lapillis, basaltic breccias...) and residual cover of red and ochre clays. Its center is characterized by volcanism occurring after a marine transgression between 1.6 and 0.9 Myrs, with volcanos imbricated into each other with different composition (labradoritic and andesitic flows versus dacitic and basaltic breccias). The East and the West of the island are composed by andesitic and labradoritic flows, andesitic breccias, andesitic pumices and pyroclastic sediments dating to Plio-Quaternary (900-550 kyrs). Two main massifs were identified in the southern part of the island. The Monts Caraïbes massif construction was initiated 500 kyrs ago by intensive effusive submarine activity. The Grande-Découverte Complex volcano construction ( [Boudon et al., 1987] and [Boudon et al., 2008]), the youngest volcanic complex of Basse-Terre was initiated $205 \mathrm{kyrs}$ ago, following three main stages: Grande-Découverte (200-42 kyrs), Carmichaël (42-11.5 kyrs) and La Soufrière (8.5 kyrs to present). 


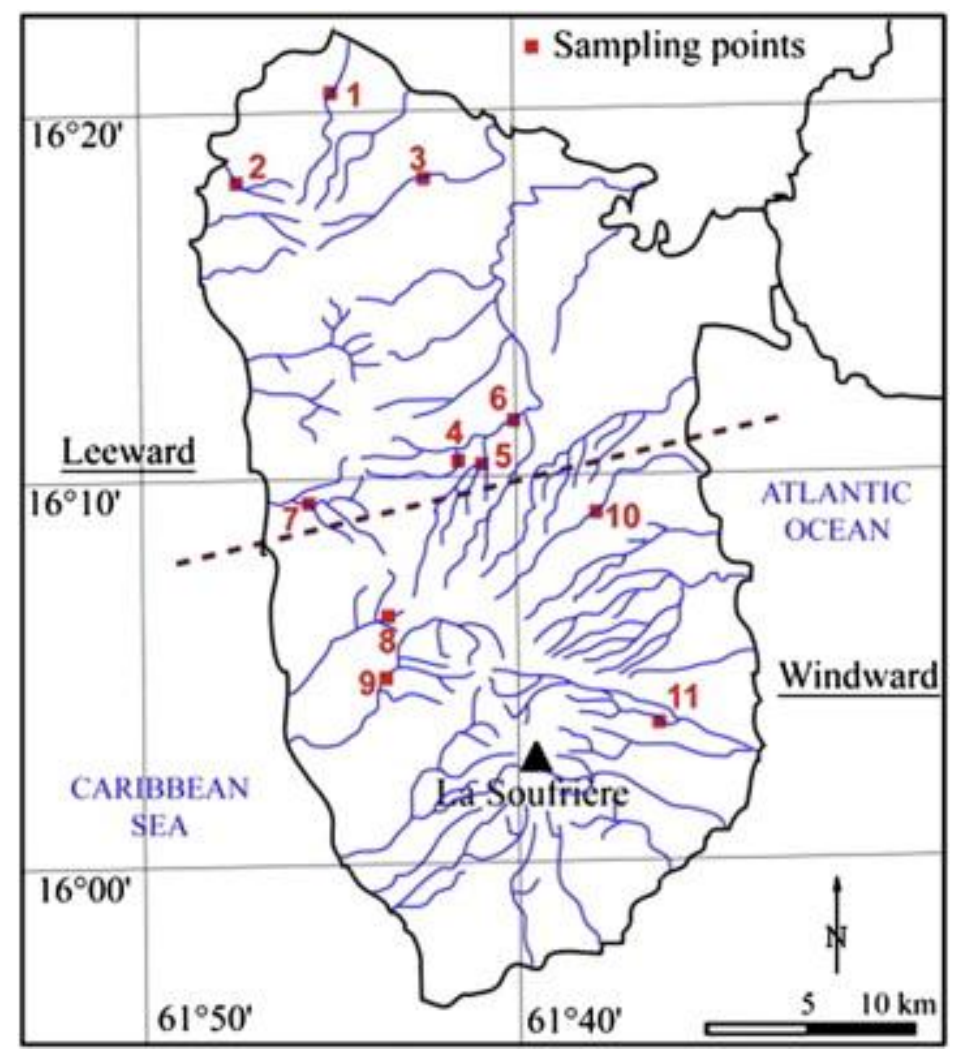

Fig. 1. Sampling location on Basse-Terre Island. The substratum ages range from 2.7 to 1.2 Myrs above the dotted line and are $\leq 900$ kyrs below this line (Samper et al., 2007).1. Desbonnes River, 2. Deshaies River, 3. Moustique Sainte-Rose River, 4. Bras-David River, 5. Corossol River, 6. Grande Rivière à Goyaves, 7. Lostau River, 8. Beaugendre River, 9. Grande Rivière de Vieux-Habitants, 10. Moustique Petit-Bourg River, 11. Grande Rivière de la Capesterre.

The Guadeloupe Island is characterized by a wet tropical climate, with a mean annual temperature and humidity around $23{ }^{\circ} \mathrm{C}$ and $75 \%$, respectively. The average annual precipitation ranges from 1200 to $8000 \mathrm{~mm} \mathrm{yr}^{-1}$, depending on the topography (the highest at the top of La Soufrière massif). The island is characterized by a wet rainy season, corresponding to the maximum of precipitations and notably extreme meteorological events (i.e. tropical storms and cyclones, Zahibo et al., 2007), from June to November, and a drier season from December to May. The spatial distribution of precipitations is controlled by the orographic effect. Thus, the East coast receives high precipitations because of the strong influence of the Easterly and conversely, the West coast, protected by the topography, receives less water.

The steep slopes and the abundant precipitations generate a dense river network flowing through the volcanic substratum of Basse-Terre Island into the Atlantic Ocean or the Caribbean Sea. The island has about 55 independent rivers with watershed areas greater than $1 \mathrm{~km}^{2}$ ( 25 on the East coast and 30 on the West coast), the largest river $\left(130 \mathrm{~km}^{2}\right)$ corresponding to the "Grande Rivière à Goyaves". Valley slopes, resulting from the younger volcanic relief, exceed $49 \%$ in the southern part of the island, while they are smaller in the northern part of the island (Plaisir et al., 2003; Table 1). In the North, the water stocks are smaller than in the Center and in the South and during the dry season most of the northern rivers do not flow. Since 1950, discharges of major rivers have been monitored first by the 
IRD (formerly the ORSTOM, French Research Institute for the Development) and now by the DIREN (French Water Survey agency) and data in Table 1 were obtained at the following web site: http://www.hydro.eaufrance.fr.

Table 1. Watersheds characteristics: sampling point geographic position (latitude and longitude), elevation above sea level, surface area and slopes of the watershed. Hydrologic characteristic: operating dates of gauging stations, discharges and runoffs during low water and flood levels calculated from DIREN data, and mean annual runoff, rainfalls and evapotranspiration.

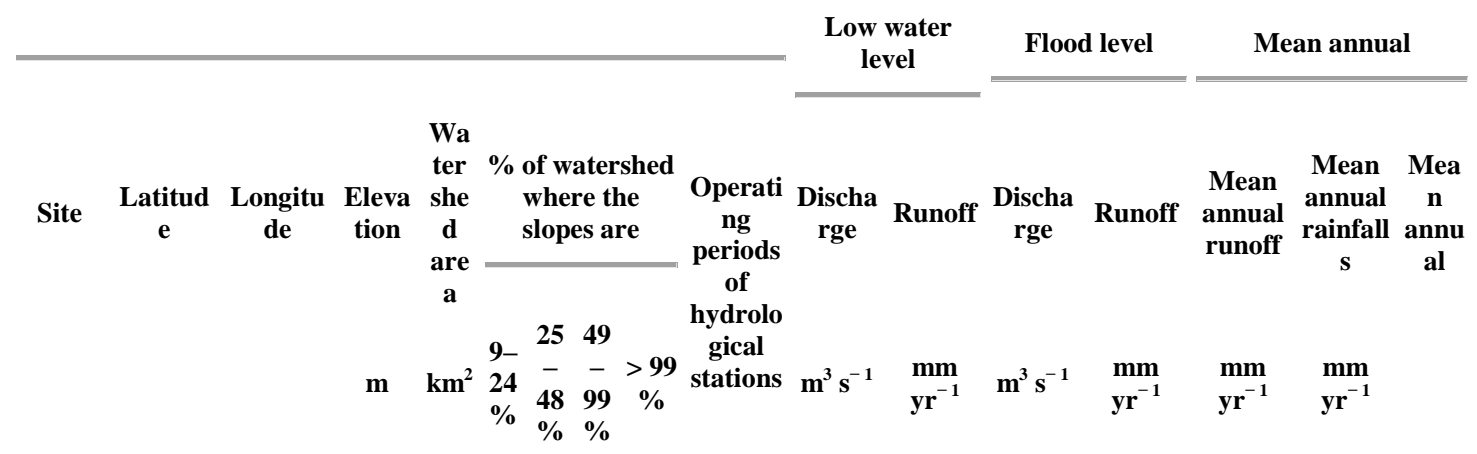

North

\begin{tabular}{|c|c|c|c|c|c|c|c|c|c|c|c|c|c|c|c|}
\hline $\begin{array}{l}\text { Desbon } \\
\text { nes }\end{array}$ & $\begin{array}{l}\mathrm{N} 16^{\circ} 19^{\prime} \\
34.4^{\prime \prime}\end{array}$ & $\begin{array}{l}\mathrm{W} 61^{\circ} 44^{\prime} \\
48.0^{\prime \prime}\end{array}$ & 136 & 5.5034 & 59 & 7 & 0 & $\begin{array}{l}1979- \\
1986\end{array}$ & $\begin{array}{l}0.07 \pm \\
0.05\end{array}$ & $\begin{array}{l}400 \pm 2 \\
90\end{array}$ & $\begin{array}{l}0.72 \pm \\
0.56\end{array}$ & $\begin{array}{l}4130 \pm 3 \\
200\end{array}$ & $\begin{array}{l}780 \pm 5 \\
80\end{array}$ & $\begin{array}{l}1790 \pm \\
450\end{array}$ & $\begin{array}{l}2.3 \pm \\
1.4\end{array}$ \\
\hline $\begin{array}{l}\text { Deshai } \\
\text { es }\end{array}$ & $\begin{array}{l}\mathrm{N} 16^{\circ} 18^{\prime} \\
05.3^{\prime \prime}\end{array}$ & $\begin{array}{l}\text { W61 } 41^{\circ} \\
29.8^{\prime \prime}\end{array}$ & 30 & 4.3846 & 50 & 4 & 0 & $\begin{array}{l}1979- \\
1982\end{array}$ & $\begin{array}{l}0.04 \pm \\
0.02\end{array}$ & $\begin{array}{l}260 \pm 1 \\
40\end{array}$ & $\begin{array}{l}0.28 \pm \\
0.16\end{array}$ & $\begin{array}{l}2030 \pm 1 \\
170\end{array}$ & $\begin{array}{l}430 \pm 2 \\
50\end{array}$ & $\begin{array}{l}1750 \pm \\
440\end{array}$ & $\begin{array}{l}4.0 \pm \\
2.1\end{array}$ \\
\hline $\begin{array}{l}\text { Mousti } \\
\text { que } \\
\text { Sainte- } \\
\text { Rose }\end{array}$ & $\begin{array}{l}\mathrm{N} 16^{\circ} 17^{\prime} \\
54.9^{\prime \prime}\end{array}$ & $\begin{array}{l}\text { W61 } 41^{\prime} \\
38.8^{\prime \prime}\end{array}$ & 114 & 6.1652 & 45 & 3 & 0 & $\begin{array}{l}1983- \\
2006\end{array}$ & $\begin{array}{l}0.19 \pm \\
0.10\end{array}$ & $\begin{array}{l}970 \pm 5 \\
30\end{array}$ & $\begin{array}{l}1.47 \pm \\
0.93\end{array}$ & $\begin{array}{l}7530 \pm 4 \\
770\end{array}$ & $\begin{array}{l}1620 \pm \\
950\end{array}$ & $\begin{array}{l}2290 \pm \\
570\end{array}$ & $\begin{array}{l}1.4 \pm \\
0.7\end{array}$ \\
\hline \multicolumn{16}{|l|}{ Center } \\
\hline $\begin{array}{l}\text { Bras- } \\
\text { David }\end{array}$ & $\begin{array}{l}\mathrm{N} 16^{\circ} 10^{\prime} \\
33.6^{\prime \prime}\end{array}$ & $\begin{array}{l}\text { W6 } 61^{\circ} 41^{\prime} \\
34.8^{\prime \prime}\end{array}$ & 233 & $\begin{array}{l}11.038 \\
0\end{array}$ & 48 & 14 & 0 & $\begin{array}{l}2001- \\
2010\end{array}$ & $\begin{array}{l}0.59 \pm \\
0.27\end{array}$ & $\begin{array}{l}1680 \pm \\
770\end{array}$ & $\begin{array}{l}3.06 \pm \\
1.27\end{array}$ & $\begin{array}{l}8770 \pm 3 \\
640\end{array}$ & $\begin{array}{l}2390 \pm \\
1050\end{array}$ & $\begin{array}{l}3410 \pm \\
850\end{array}$ & $\begin{array}{l}1.4 \pm \\
0.6\end{array}$ \\
\hline $\begin{array}{l}\text { Coross } \\
\text { ol }\end{array}$ & $\begin{array}{l}\mathrm{N} 16^{\circ} 10^{\prime} \\
48.8^{\prime \prime}\end{array}$ & $\begin{array}{l}\mathrm{W} 61^{\circ} 40^{\prime} \\
49.5^{\prime \prime}\end{array}$ & 179 & $\begin{array}{l}12.5 \\
2\end{array}$ & 33 & 26 & 2 & & & $\begin{array}{l}1580 \pm \\
940\end{array}$ & & $\begin{array}{l}10510 \pm \\
4690\end{array}$ & & & \\
\hline $\begin{array}{l}\text { Goyave } \\
\mathrm{s}\end{array}$ & $\begin{array}{l}\mathrm{N} 16^{\circ} 11^{\prime} \\
27.7^{\prime \prime}\end{array}$ & $\begin{array}{l}\text { W61 } 39^{\prime} \\
33.0^{\prime \prime}\end{array}$ & 125 & $\begin{array}{ll}14.4 & 48 \\
0 & \end{array}$ & 28 & 23 & 1 & $\begin{array}{l}1973- \\
2002\end{array}$ & $\begin{array}{l}0.68 \pm \\
0.51\end{array}$ & $\begin{array}{l}1480 \pm \\
1120\end{array}$ & $\begin{array}{l}5.59 \pm \\
2.61\end{array}$ & $\begin{array}{l}12240 \pm \\
5730\end{array}$ & $\begin{array}{l}2560 \pm \\
1580\end{array}$ & $\begin{array}{l}3450 \pm \\
860\end{array}$ & $\begin{array}{l}1.3 \pm \\
0.7\end{array}$ \\
\hline \multicolumn{16}{|l|}{ West } \\
\hline Lostau & $\begin{array}{l}\mathrm{N} 16^{\circ} 09^{\prime} \\
34.1^{\prime \prime}\end{array}$ & $\begin{array}{l}\text { W6 } 61^{\circ} 45^{\prime} \\
35.9^{\prime \prime}\end{array}$ & 108 & 8.049 & 41 & 49 & 1 & $\begin{array}{l}1979- \\
1987\end{array}$ & $\begin{array}{l}0.29 \pm \\
0.16\end{array}$ & $\begin{array}{l}1130 \pm \\
620\end{array}$ & $\begin{array}{l}1.71 \pm \\
0.78\end{array}$ & $\begin{array}{l}6710 \pm 3 \\
070\end{array}$ & $\begin{array}{l}1690 \pm \\
860\end{array}$ & $\begin{array}{l}2740 \pm \\
680\end{array}$ & $\begin{array}{l}1.6 \pm \\
0.7\end{array}$ \\
\hline $\begin{array}{l}\text { Beauge } \\
\text { ndre }\end{array}$ & $\begin{array}{l}\mathrm{N} 16^{\circ} 06^{\prime} \\
27.6^{\prime \prime}\end{array}$ & $\begin{array}{l}\text { W61 } 44^{\prime} \\
09.1^{\prime \prime}\end{array}$ & 225 & 8.176 & 37 & 53 & 4 & $\begin{array}{l}1979- \\
1991\end{array}$ & $\begin{array}{l}0.31 \pm \\
0.26\end{array}$ & $\begin{array}{l}1190 \pm \\
990\end{array}$ & $\begin{array}{l}2.71 \pm \\
1.32\end{array}$ & $\begin{array}{l}10450 \pm \\
5100\end{array}$ & $\begin{array}{l}2120 \pm \\
1400\end{array}$ & $\begin{array}{l}2720 \pm \\
680\end{array}$ & $\begin{array}{l}1.3 \pm \\
0.7\end{array}$ \\
\hline $\begin{array}{l}\text { Vieux- } \\
\text { Habita } \\
\text { nts }\end{array}$ & $\begin{array}{l}\mathrm{N} 16^{\circ} 05^{\prime} \\
11.8^{\prime \prime}\end{array}$ & $\begin{array}{l}\text { W61 } 41^{\circ} \\
31.3^{\prime \prime}\end{array}$ & 257 & $\begin{array}{ll}19.1 & 13 \\
0 & \end{array}$ & 32 & 51 & 4 & $\begin{array}{l}1980- \\
2009\end{array}$ & $\begin{array}{l}1.50 \pm \\
0.90\end{array}$ & $\begin{array}{l}2480 \pm \\
1490\end{array}$ & $\begin{array}{l}9.81 \pm \\
4.18\end{array}$ & $\begin{array}{l}16190 \pm \\
6900\end{array}$ & $\begin{array}{l}3850 \pm \\
2030\end{array}$ & $\begin{array}{l}3830 \pm \\
960\end{array}$ & $\begin{array}{l}1.0 \pm \\
0.5\end{array}$ \\
\hline \multicolumn{16}{|l|}{ East } \\
\hline $\begin{array}{l}\text { Mousti } \\
\text { que } \\
\text { Petit- } \\
\text { Bourg }\end{array}$ & $\begin{array}{l}\mathrm{N} 16^{\circ} 09^{\prime} \\
41.8^{\prime \prime}\end{array}$ & $\begin{array}{l}\text { W61 } 37^{\circ} \\
37.8^{\prime \prime}\end{array}$ & 100 & $\begin{array}{ll}11.5 & \\
0 & \end{array}$ & 41 & 31 & 1 & $\begin{array}{l}1984- \\
2010\end{array}$ & $\begin{array}{l}0.72 \pm \\
0.42\end{array}$ & $\begin{array}{l}1970 \pm \\
1150\end{array}$ & $\begin{array}{l}4.44 \pm \\
1.85\end{array}$ & $\begin{array}{l}12160 \pm \\
5080\end{array}$ & $\begin{array}{l}2990 \pm \\
1550\end{array}$ & $\begin{array}{l}3990 \pm \\
1000\end{array}$ & $\begin{array}{l}1.3 \pm \\
0.6\end{array}$ \\
\hline $\begin{array}{l}\text { Capest } \\
\text { erre }\end{array}$ & $\begin{array}{l}\mathrm{N} 16^{\circ} 04^{\prime} \\
18.0^{\prime \prime}\end{array}$ & $\begin{array}{l}\text { W61 } 36^{\circ} \\
34.1^{\prime \prime}\end{array}$ & 208 & $\begin{array}{ll}16.2 & 13 \\
0 & \end{array}$ & 32 & 45 & 5 & $\begin{array}{l}1983- \\
2010\end{array}$ & & & & & & & \\
\hline
\end{tabular}


The climatic conditions promote the growth of abundant vegetation on Basse-Terre. The vegetation cover depends both on the meteorological conditions and the elevation (Rousteau, 1996). The vegetation on the island is represented by four levels from lower altitudes to higher altitudes: 1) a semi-deciduous forest on volcanic terrain, 2) a seasonal evergreen forest, 3) a mountain and sub-mountain rainforest and, 4) an altimountain forest.

Different soil types have developed on the volcanic andesitic basement, depending on rock age, precipitations and topography ( [Colmet-Daage, 1969], [Colmet-Daage and Bernard, 1979] and [Buss et al., 2010]). In the North, vertisols are characterized by high contents of montmorillonite and kaolinite typical of such old formations under tropical climate. The central part of the island is characterized by ferralitic soils with halloysites and iron oxides. East, West and South are characterized by andosols with allophanes, typical of volcanic rocks and wet tropical regions. Locally paleosols may occur.

The choice of the rivers was govern by their geological substratum, the pedology and the exposure to rainfalls. The water samples were collected during dry and wet seasons from 2006 to 2008, in order to assess the influence of the hydrological regime on surface water chemistry. In the South of the Basse-Terre, rivers were not sampled, because their chemistry is influenced by hydrothermal inputs, due to the Soufrière volcanic activity. They are characterized by high sulfate and cations concentrations (ex. Rivière Noire, Rad et al., 2007) and are not all perennial. This study focuses on 11 rivers located in the North $(n=3)$, the Center $(n=3)$, the West coast $(n=3)$ and the East coast $(n=2)$ (Fig. 1). The northern watersheds are characterized by less steep slopes (slope < 49\%), rock older than $1 \mathrm{Myr}$ and thick ferralitic soils and weak rainfalls; center watersheds have quite the same characteristics but higher rainfalls; western watersheds are characterized by steeper slopes (slope $>49 \%$ ), an age less than 800 kyrs, thin andosoils, weak rainfalls; eastern watersheds have also steep slopes, an age less than $800 \mathrm{kyrs}$, thin andosoils and are subject to strong rainfalls.

\section{Sampling and analytical methods}

\subsection{Hydrological data and calculation of rates and yields}

The discharge was measured at gauging stations installed by the IRD or more recently by the DIREN. The water level is measured at gauging stations with a pressure detector. Gauging campaigns are regularly conducted to adjust the calibration curves relating discharge with water level (i.e. given by gauging scale). The different discharge values are available at: http://www.hydro.eaufrance.fr.

As some rivers are no longer equipped by gauging station (Table 1) discharge data at the time of sampling for all rivers is not available. We need however discharge values to calculate the riverine export rates. Based on the DIREN discharge dataset, we estimated, for each river a mean discharge value for low water and flood conditions. In Fig. 2, representing the discharge versus frequency for the Bras-David River, two hydrological periods are evidenced. The first part (before the break), corresponding to $90 \%$ of the annual flux is characteristic of low water level. The second part (after the break), corresponding to $10 \%$ of the annual flux, is characteristic of flood conditions. The distribution between both hydrological conditions is the same for all rivers, even if mean daily values vary from one river to the other. For rivers where present day gauging data were not available, their average discharge values corresponding to low water and flood conditions were calculated with the help of historical records of the DIREN. 


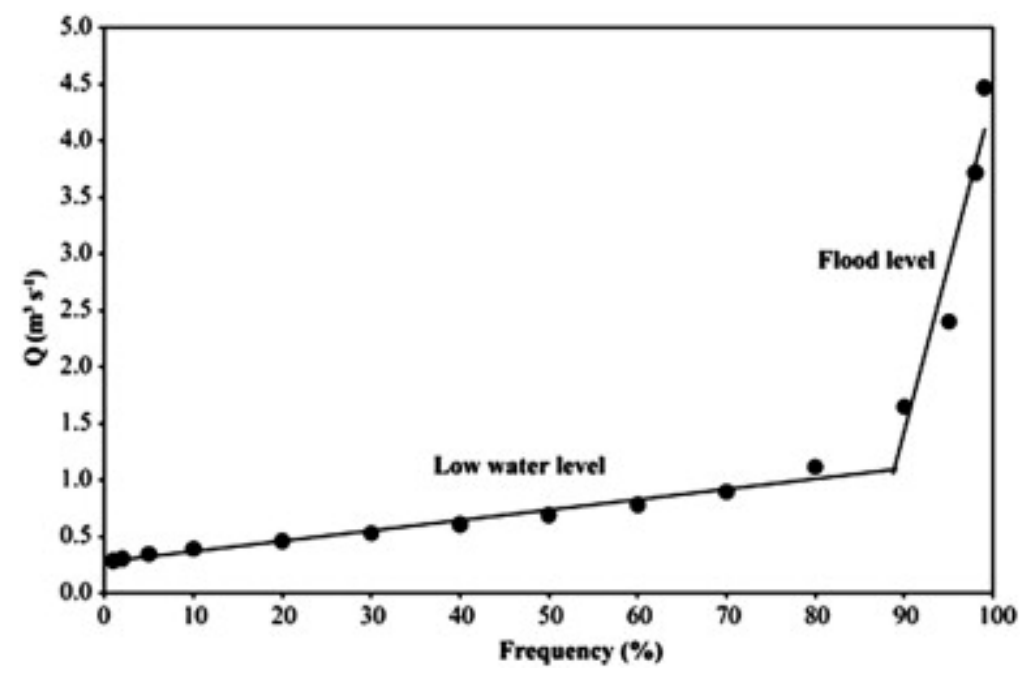

Fig. 2. Plot of frequency vs discharge $\left(\mathrm{m}^{3} \mathrm{~s}^{-1}\right)$ for the Bras-David River (ORSTOM-IRD and DIREN data, Table 1). The lines represent the fits for the low water and flood periods. The flood regimes represent around $10 \%$ of the annual discharge. This trend is observed for all Guadeloupean rivers.

The runoff (in $\mathrm{mm} \mathrm{yr}^{-1}$ ) is defined by the following formula:

Runoff $=\frac{\text { Discharge }}{\text { Area }_{\text {watershed }}}$

The mean annual runoff is calculated with the following formula:

mean annual runoff $=R L W L$ [?] $0.9+R F L$ ?? 0.1

with $\mathrm{R}_{\mathrm{LWL}}=$ mean runoff during low water level, and $\mathrm{R}_{\mathrm{FL}}=$ mean runoff during floods

The associated errors have been calculated with the minimal and maximal values during low water level or flood level. Using the rainfalls data from French meteorological survey agency (Météo-France), we estimated the mean annual rainfalls for each watershed. The mean annual evapotranspiration factor is the calculated ratio between rainfalls and runoff.

\subsection{Sample collection}

Pristine water samples were collected, upstream of the anthropogenic activities like the sugar cane and banana crops (Fig. 1). The sampling of surface water was done manually from 2006 to 2008 at different hydrological stages corresponding to low water levels (LWL) and flood levels (FL). Water samples used for the measurement of dissolved major ions concentrations were filtered through $0.2 \mu \mathrm{m}$ cellulose acetate filters. For cations measurements, samples were acidified to $\mathrm{pH}=2$ with distilled nitric acid. Non-acidified samples were stored at $4{ }^{\circ} \mathrm{C}$ in the dark for the measurements of anions and dissolved silica concentrations. Samples used for the measurement of dissolved organic carbon concentrations and $\delta^{13} \mathrm{C}$ were filtered through glass fiber filters $\left(\mathrm{GF} / \mathrm{F}\right.$ Whatman ${ }^{\circledR}$ by Schleicher $\&$ Schuell cut off $\left.0.7 \mu \mathrm{m}\right)$ acidified with concentrated $\mathrm{H}_{3} \mathrm{PO}_{4}$ in pre-cleaned and pre-burned Grasse amber glass bottles and stored at $4{ }^{\circ} \mathrm{C}$ in the dark. A few sampled $(\mathrm{n}=25)$ were collected in 2008 for the measurements of $\delta^{13} \mathrm{C}$ of dissolved inorganic carbon in sealed glass vials. 


\subsection{Analytical methods}

Temperature, $\mathrm{pH}$ and conductivity were measured in the field (Table 2). The precision of $\mathrm{pH}$ measurements was $0.05 \mathrm{pH}$ units. Conductivity was measured with a Hanna HI9835 probe. The precision was $1 \%$. Alkalinity values were measured in the laboratory with an automatic acid-base titration stand (Radiometer TIM840 with Schott probe) by the Gran method with a precision of $1 \%$.

Table 2. Physico-chemical parameters and organic matter characteristics of water samples recovered on the Basse-Terre during low water levels and flood levels, *data calculated, NICB (Normalized Ionic Charge Balance), TDScat and TDSw.

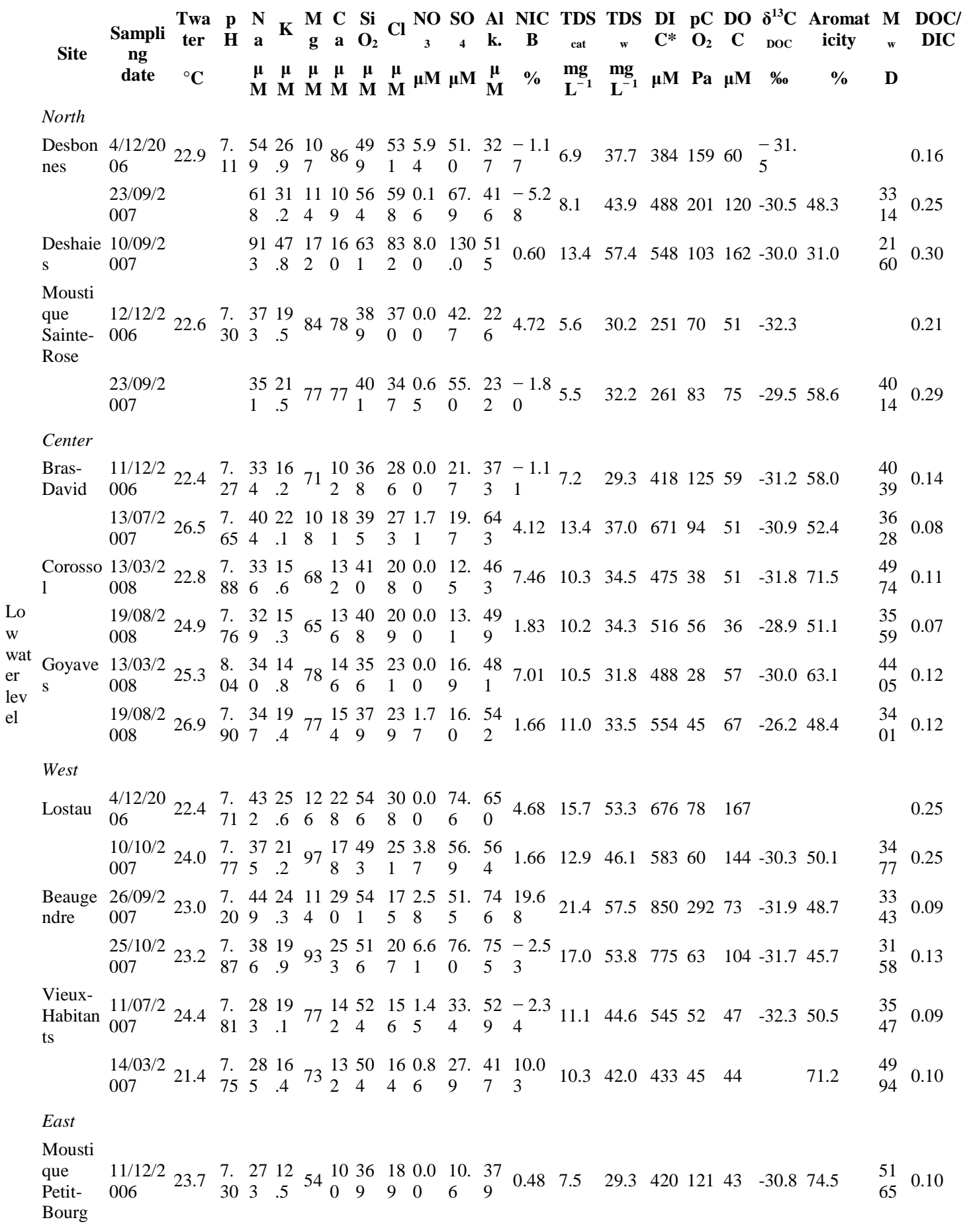




\begin{tabular}{|c|c|c|c|c|c|c|c|c|c|c|c|c|c|c|c|c|c|c|c|c|c|c|}
\hline Site & $\begin{array}{c}\text { Sampli } \\
\text { ng } \\
\text { date }\end{array}$ & $\begin{array}{c}\text { Twa } \\
\text { ter } \\
\\
{ }^{\circ} \mathbf{C}\end{array}$ & $\begin{array}{l}\mathbf{p} \\
\mathbf{H}\end{array}$ & $\begin{array}{l}\mathbf{N} \\
\mathbf{a} \\
\mu \\
\mathbf{M}\end{array}$ & $\begin{array}{l}\mathbf{K} \\
\boldsymbol{\mu} \\
\mathbf{M}\end{array}$ & $\begin{array}{c}\text { M } \\
\mathbf{g} \\
\boldsymbol{\mu} \\
\mathbf{M}\end{array}$ & & $\begin{array}{l}\mathrm{Si} \\
\mathbf{O}_{2} \\
\boldsymbol{\mu} \\
\mathbf{M}\end{array}$ & $\begin{array}{l}\mathrm{Cl} \\
\\
\mu \\
\mathbf{M}\end{array}$ & $\begin{array}{c}\text { NO } \\
3 \\
\mu M\end{array}$ & $\begin{array}{c}\text { SO } \\
4 \\
\mu M\end{array}$ & $\begin{array}{l}\text { Al } \\
\text { k. } \\
\mu \\
\text { M }\end{array}$ & $\begin{array}{c}\text { NIC } \\
\text { B } \\
\%\end{array}$ & $\begin{array}{c}\text { TDS } \\
\text { cat } \\
\mathbf{m g} \\
\mathbf{L}^{-1}\end{array}$ & $\begin{array}{c}\text { TDS } \\
\text { w } \\
\mathbf{m g}^{-1} \\
\mathbf{L}^{-1}\end{array}$ & $\begin{array}{l}\text { DI } \\
\mathbf{C}^{*} \\
\boldsymbol{\mu M}\end{array}$ & $\begin{array}{c}\mathbf{p C} \\
\mathrm{O}_{2} \\
\mathrm{~Pa}\end{array}$ & $\begin{array}{c}\text { DO } \\
\text { C } \\
\mu \mathrm{M}\end{array}$ & $\begin{array}{c}\delta^{13} \mathrm{C} \\
\text { Doc } \\
\%\end{array}$ & $\begin{array}{c}\text { Aromat } \\
\text { icity } \\
\%\end{array}$ & $\begin{array}{l}\text { M } \\
\text { w } \\
\text { D }\end{array}$ & $\begin{array}{c}\text { DOC/ } \\
\text { DIC }\end{array}$ \\
\hline & $\begin{array}{l}8 / 11 / 20 \\
07\end{array}$ & & $\begin{array}{l}7 . \\
31\end{array}$ & $\begin{array}{l}27 \\
6\end{array}$ & $\begin{array}{l}13 \\
.0\end{array}$ & $60 \frac{1}{1}$ & $\begin{array}{l}12 \\
1\end{array}$ & $\begin{array}{l}44 \\
9\end{array}$ & $\begin{array}{l}16 \\
6\end{array}$ & $\begin{array}{l}0.8 \\
1\end{array}$ & 9.9 & $\begin{array}{l}49 \\
4\end{array}$ & $\begin{array}{l}-5.0 \\
1\end{array}$ & 9.2 & 35.8 & 547 & 153 & 54 & -32.8 & 57.2 & $\begin{array}{l}39 \\
61\end{array}$ & 0.10 \\
\hline \multirow[t]{2}{*}{$\begin{array}{l}\text { Capeste } \\
\text { rre }\end{array}$} & $\begin{array}{l}9 / 10 / 20 \\
07\end{array}$ & 23.7 & $\begin{array}{l}7 . \\
70\end{array}$ & $\begin{array}{l}23 \\
5\end{array}$ & $\begin{array}{l}15 \\
.1\end{array}$ & $56 \frac{1}{6}$ & & $\begin{array}{l}11 \\
0\end{array}$ & $\begin{array}{l}14 \\
7\end{array}$ & & $\begin{array}{l}23 . \\
2\end{array}$ & $\begin{array}{l}42 \\
9\end{array}$ & $\begin{array}{l}-2.1 \\
7\end{array}$ & 8.9 & 16.6 & 447 & 54 & 46 & -32.7 & 65.8 & $\begin{array}{l}45 \\
93\end{array}$ & 0.10 \\
\hline & $\begin{array}{l}18 / 06 / 2 \\
008\end{array}$ & 24.2 & $\begin{array}{l}7 . \\
91\end{array}$ & $\begin{array}{l}26 \\
8 .\end{array}$ & $\begin{array}{l}15 \\
.1\end{array}$ & $58 \frac{1}{9}$ & $\begin{array}{l}12 \\
9\end{array}$ & $\begin{array}{l}43 \\
6\end{array}$ & $\begin{array}{l}14 \\
4\end{array}$ & $\begin{array}{l}0.0 \\
0\end{array}$ & $\begin{array}{l}25 . \\
0\end{array}$ & $\begin{array}{l}43 \\
9\end{array}$ & 3.99 & 9.9 & 37.4 & 449 & 34 & 54 & -28.4 & 49.1 & $\begin{array}{l}33 \\
96\end{array}$ & 0.12 \\
\hline \multicolumn{23}{|l|}{ North } \\
\hline \multirow[t]{2}{*}{$\begin{array}{l}\text { Deshaie } \\
\mathrm{s}\end{array}$} & $\begin{array}{l}23 / 09 / 2 \\
007\end{array}$ & & & $\begin{array}{l}54 \\
0\end{array}$ & $\begin{array}{l}42 \\
.7\end{array}$ & 778 & & & $\begin{array}{l}40 \\
3\end{array}$ & $\begin{array}{l}41 . \\
00\end{array}$ & $\begin{array}{l}72 . \\
0\end{array}$ & $\begin{array}{l}28 \\
6\end{array}$ & 3.34 & 9.6 & 13.4 & 305 & 58 & 601 & -27.2 & 53.8 & $\begin{array}{l}36 \\
88\end{array}$ & 1.97 \\
\hline & $\begin{array}{l}9 / 10 / 20 \\
08\end{array}$ & 26.1 & $\begin{array}{l}7 . \\
51\end{array}$ & $\begin{array}{l}64 . \\
9 .\end{array}$ & $\begin{array}{l}37 \\
.9\end{array}$ & 998 & & $\begin{array}{l}38 \\
4\end{array}$ & $\begin{array}{l}52 \\
7\end{array}$ & $\begin{array}{l}5.8 \\
1\end{array}$ & $\begin{array}{l}86 . \\
4\end{array}$ & $\begin{array}{l}27 \\
8\end{array}$ & 7.05 & 9.5 & 36.9 & 295 & 56 & 332 & & 72.5 & $\begin{array}{l}49 \\
60\end{array}$ & 1.12 \\
\hline $\begin{array}{l}\text { Mousti } \\
\text { que } \\
\text { Sainte- } \\
\text { Rose }\end{array}$ & $\begin{array}{l}9 / 10 / 20 \\
08\end{array}$ & 23.7 & $\begin{array}{l}7 . \\
19\end{array}$ & $\begin{array}{l}29 \\
8 .\end{array}$ & $\begin{array}{l}18 \\
.7\end{array}$ & 606 & & $\begin{array}{l}27 \\
4\end{array}$ & $\begin{array}{l}25 \\
6\end{array}$ & $\begin{array}{l}0.0 \\
0\end{array}$ & $\begin{array}{l}44 . \\
2\end{array}$ & $\begin{array}{l}21 \\
2\end{array}$ & 0.65 & 5.3 & 24.1 & 242 & 87 & 281 & & 36.2 & $\begin{array}{l}25 \\
21\end{array}$ & 1.16 \\
\hline \multicolumn{23}{|l|}{ Center } \\
\hline \multirow[t]{2}{*}{$\begin{array}{l}\text { Bras- } \\
\text { David }\end{array}$} & $\begin{array}{l}27 / 09 / 2 \\
007\end{array}$ & & $\begin{array}{l}7 . \\
24\end{array}$ & $\begin{array}{l}24 \\
3\end{array}$. & $\begin{array}{l}14 \\
.1\end{array}$ & 496 & & $\begin{array}{l}24 \\
2\end{array}$ & $\begin{array}{l}19 \\
6\end{array}$ & $\begin{array}{l}0.1 \\
6\end{array}$ & $\begin{array}{l}24 . \\
6\end{array}$ & $\begin{array}{l}22 \\
7\end{array}$ & 2.65 & 5.3 & 20.7 & 255 & 84 & 168 & -29.4 & 48.2 & $\begin{array}{l}33 \\
22\end{array}$ & 0.66 \\
\hline & $\begin{array}{l}9 / 10 / 20 \\
08\end{array}$ & & $\begin{array}{l}7 . \\
48\end{array}$ & $\begin{array}{l}21 \\
8 .\end{array}$ & $\begin{array}{l}12 \\
.8\end{array}$ & 549 & & $\begin{array}{l}27 \\
5\end{array}$ & $\begin{array}{l}16 \\
5\end{array}$ & $\begin{array}{l}0.0 \\
0\end{array}$ & $\begin{array}{l}16 . \\
3\end{array}$ & $\begin{array}{l}34 \\
3\end{array}$ & $\begin{array}{l}-1.0 \\
6\end{array}$ & 6.8 & 23.6 & 368 & 73 & 87 & & & & 0.32 \\
\hline \multirow[t]{2}{*}{$\begin{array}{l}\text { Corosso } \\
1\end{array}$} & $\begin{array}{l}10 / 09 / 2 \\
007\end{array}$ & 20.9 & $\begin{array}{l}8 . \\
10\end{array}$ & $\begin{array}{l}20 \\
1 .\end{array}$ & $\begin{array}{l}11 \\
.7\end{array}$ & 408 & & $\begin{array}{l}27 \\
1\end{array}$ & $\begin{array}{l}14 \\
7\end{array}$ & $\begin{array}{l}1.2 \\
9\end{array}$ & $\begin{array}{l}16 . \\
0\end{array}$ & $\begin{array}{l}31 \\
1\end{array}$ & $\begin{array}{l}-6.1 \\
7\end{array}$ & 6.0 & 22.7 & 315 & 15 & 239 & -29.5 & 40.9 & $\begin{array}{l}28 \\
56\end{array}$ & 0.78 \\
\hline & $\begin{array}{l}10 / 10 / 2 \\
007\end{array}$ & 23.8 & $\begin{array}{l}7 . \\
49\end{array}$ & $\begin{array}{l}22 \\
9 .\end{array}$ & $\begin{array}{l}11 \\
.8\end{array}$ & 448 & 8 & $\begin{array}{l}28 \\
8\end{array}$ & $\begin{array}{l}14 \\
7\end{array}$ & $\begin{array}{l}0.9 \\
7\end{array}$ & $\begin{array}{l}13 . \\
8\end{array}$ & $\begin{array}{l}29 \\
9\end{array}$ & 3.82 & 6.6 & 24.1 & 320 & 62 & 164 & -29.3 & 49.1 & $\begin{array}{l}34 \\
05\end{array}$ & 0.51 \\
\hline \multirow[t]{2}{*}{$\begin{array}{l}\text { Goyave } \\
\mathrm{s}\end{array}$} & $\begin{array}{l}10 / 10 / 2 \\
007\end{array}$ & 25.2 & $\begin{array}{l}7 . \\
46\end{array}$ & $\begin{array}{l}22 \\
5\end{array}$ & $\begin{array}{l}12 \\
.8\end{array}$ & 458 & & $\begin{array}{l}25 \\
6\end{array}$ & $\begin{array}{l}16 \\
0\end{array}$ & $\begin{array}{l}1.2 \\
9\end{array}$ & $\begin{array}{l}19 . \\
5\end{array}$ & $\begin{array}{l}28 \\
1\end{array}$ & 4.12 & 6.4 & 22.5 & 301 & 63 & 169 & -29.9 & 50.0 & $\begin{array}{l}34 \\
79\end{array}$ & 0.56 \\
\hline & $\begin{array}{l}20 / 06 / 2 \\
008\end{array}$ & 24.4 & $\begin{array}{l}7 . \\
17\end{array}$ & $\begin{array}{l}22 \\
5\end{array}$ & $\begin{array}{l}15 \\
.5\end{array}$ & $50 \frac{1}{2}$ & $\begin{array}{l}10 \\
2\end{array}$ & $\begin{array}{l}30 \\
1\end{array}$ & $\begin{array}{l}19 \\
5\end{array}$ & $\begin{array}{l}0.0 \\
0\end{array}$ & $\begin{array}{l}17 . \\
2\end{array}$ & $\begin{array}{l}38 \\
8\end{array}$ & $\begin{array}{l}-14 . \\
28\end{array}$ & 6.3 & 24.5 & 445 & 169 & 118 & -28.5 & 48.3 & $\begin{array}{l}33 \\
70\end{array}$ & 0.28 \\
\hline \multicolumn{23}{|l|}{ West } \\
\hline \multirow[t]{2}{*}{$\begin{array}{l}\text { Beauge } \\
\text { ndre }\end{array}$} & $\begin{array}{l}10 / 09 / 2 \\
007\end{array}$ & 23.6 & $\begin{array}{l}7 . \\
78\end{array}$ & $\begin{array}{l}33 \\
0\end{array}$ & $\begin{array}{l}23 \\
.6\end{array}$ & $72 \stackrel{1}{0}$ & $\begin{array}{l}19 \\
0\end{array}$ & $\begin{array}{l}39 \\
0\end{array}$ & $\begin{array}{l}26 \\
3\end{array}$ & $\begin{array}{l}53 . \\
87\end{array}$ & $\begin{array}{l}50 . \\
0\end{array}$ & $\begin{array}{l}54 \\
0\end{array}$ & $\begin{array}{l}-9.6 \\
7\end{array}$ & 11.5 & 37.7 & 558 & 56 & 328 & -28.7 & 48.2 & $\begin{array}{l}33 \\
23\end{array}$ & 0.60 \\
\hline & $\begin{array}{l}10 / 10 / 2 \\
007\end{array}$ & 23.0 & $\begin{array}{l}7 . \\
86\end{array}$ & $\begin{array}{l}36 \\
3\end{array}$ & $\begin{array}{l}19 \\
.4\end{array}$ & $84 \frac{2}{7}$ & $\begin{array}{l}22 \\
7\end{array}$ & $\begin{array}{l}44 \\
9\end{array}$ & $\begin{array}{l}19 \\
2\end{array}$ & $\begin{array}{l}7.4 \\
2\end{array}$ & $\begin{array}{l}66 . \\
0\end{array}$ & $\begin{array}{l}64 \\
5\end{array}$ & 2.30 & 15.6 & 47.4 & 663 & 55 & 166 & -30.1 & 44.1 & $\begin{array}{l}30 \\
78\end{array}$ & 0.25 \\
\hline \multirow[t]{2}{*}{$\begin{array}{l}\text { Vieux- } \\
\text { Habitan } \\
\text { ts }\end{array}$} & $\begin{array}{l}5 / 07 / 20 \\
07\end{array}$ & 23.3 & $\begin{array}{l}7 . \\
50\end{array}$ & $\begin{array}{l}20 \\
4\end{array}$ & $\begin{array}{l}14 \\
.2\end{array}$ & 498 & 89 & $\begin{array}{l}32 \\
4\end{array}$ & $\begin{array}{l}15 \\
1\end{array}$ & $\begin{array}{l}2.4 \\
0\end{array}$ & $\begin{array}{l}27 . \\
3\end{array}$ & $\begin{array}{l}29 \\
7\end{array}$ & $\begin{array}{l}-3.0 \\
7\end{array}$ & 6.4 & 27.3 & 318 & 59 & 131 & -29.7 & 39.2 & $\begin{array}{l}27 \\
46\end{array}$ & 0.41 \\
\hline & $\begin{array}{l}10 / 10 / 2 \\
007\end{array}$ & 23.0 & $\begin{array}{l}7 . \\
90\end{array}$ & $\begin{array}{l}19 \\
7 .\end{array}$ & $\begin{array}{l}14 \\
.1\end{array}$ & 478 & & $\begin{array}{l}34 \\
0\end{array}$ & $\begin{array}{l}10 \\
1\end{array}$ & $\begin{array}{l}1.7 \\
7\end{array}$ & $\begin{array}{l}21 . \\
9\end{array}$ & $\begin{array}{l}34 \\
4\end{array}$ & $\begin{array}{l}-3.7 \\
8\end{array}$ & 7.2 & 29.0 & 353 & 27 & 149 & -29.9 & 39.5 & $\begin{array}{l}27 \\
70\end{array}$ & 0.42 \\
\hline \multicolumn{23}{|l|}{ East } \\
\hline \multirow[t]{2}{*}{$\begin{array}{l}\text { Mousti } \\
\text { que } \\
\text { Petit- } \\
\text { Bourg }\end{array}$} & $\begin{array}{l}28 / 03 / 2 \\
008\end{array}$ & & $\begin{array}{l}6 . \\
72\end{array}$ & $\begin{array}{l}23 \\
3\end{array}$ & $\begin{array}{l}12 \\
.0\end{array}$ & 468 & & $\begin{array}{l}24 \\
7\end{array}$ & $\begin{array}{l}19 \\
1\end{array}$ & $\begin{array}{l}0.0 \\
0\end{array}$ & $\begin{array}{l}12 . \\
9\end{array}$ & $\begin{array}{l}24 \\
5\end{array}$ & 8.26 & 5.7 & 20.3 & 349 & 298 & 109 & -29.7 & 44.0 & $\begin{array}{l}30 \\
43\end{array}$ & 0.31 \\
\hline & $\begin{array}{l}9 / 10 / 20 \\
08\end{array}$ & 23.7 & $\begin{array}{l}7 . \\
19\end{array}$ & $\begin{array}{l}12 \\
0\end{array}$. & $\begin{array}{l}11 \\
.3\end{array}$ & 194 & & $\begin{array}{l}12 \\
7\end{array}$ & 72 & $\begin{array}{l}3.7 \\
1\end{array}$ & $\begin{array}{l}11 . \\
6\end{array}$ & $\begin{array}{l}21 \\
2\end{array}$ & $\begin{array}{l}-21 \\
34\end{array}$ & 3.7 & 11.8 & 243 & 88 & 227 & -28.8 & 58.0 & $\begin{array}{l}39 \\
67\end{array}$ & 0.93 \\
\hline \multirow[t]{2}{*}{$\begin{array}{l}\text { Capeste } \\
\text { rre }\end{array}$} & $\begin{array}{l}27 / 09 / 2 \\
007\end{array}$ & & $\begin{array}{l}7 . \\
06\end{array}$ & $\begin{array}{l}14 \\
0\end{array}$. & $\begin{array}{l}10 \\
.7\end{array}$ & 265 & & $\begin{array}{l}16 \\
0\end{array}$ & $\begin{array}{l}10 \\
9\end{array}$ & $\begin{array}{l}1.4 \\
5\end{array}$ & $\begin{array}{l}16 . \\
9\end{array}$ & $\begin{array}{l}14 \\
8\end{array}$ & 6.61 & 3.8 & 14.2 & 176 & 82 & 236 & -28.4 & 38.3 & $\begin{array}{l}26 \\
63\end{array}$ & 1.34 \\
\hline & $\begin{array}{l}27 / 11 / 2 \\
007\end{array}$ & 21.8 & $\begin{array}{l}7 . \\
07\end{array}$ & $\begin{array}{l}18 \\
8 .\end{array}$ & $\begin{array}{l}12 \\
.0\end{array}$ & 428 & 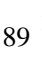 & $\begin{array}{l}24 \\
6\end{array}$ & $\begin{array}{l}14 \\
9\end{array}$ & $\begin{array}{l}0.0 \\
0\end{array}$ & $\begin{array}{l}24 . \\
9\end{array}$ & $\begin{array}{l}21 \\
8\end{array}$ & 9.79 & 5 & & & & & & & & \\
\hline
\end{tabular}


Laboratory measurements of major ions concentrations were done by ionic chromatography (Dionex DX120, precision 5\%). The detection limits for $\mathrm{Ca}, \mathrm{Mg}, \mathrm{Na}$ and $\mathrm{K}$ were respectively $12.5 \mu \mathrm{M}, 20.6 \mu \mathrm{M}, 0.9 \mu \mathrm{M}$ and $0.5 \mu \mathrm{M}$. The detection limits for $\mathrm{SO}_{4}, \mathrm{Cl}, \mathrm{NO}_{3}$ and $\mathrm{F}$ were respectively $4.2 \mu \mathrm{M}, 8.5 \mu \mathrm{M}, 3.2 \mu \mathrm{M}$ and $5.2 \mu \mathrm{M}$. Dissolved silica concentrations were determined calorimetrically, with a Seal QUAATRO analyzer (detection limit $5 \mu \mathrm{M}$ and precision $2 \%$ ).

Dissolved organic carbon (DOC) concentrations were measured using a Shimadzu TOCVCSH analyzer (Sugimura and Suzuki, 1988). The detection limit was $20 \mu \mathrm{M}$ and the precision was $2 \%$. The $\delta^{13} \mathrm{C}_{\mathrm{DOC}}$ was measured at the "Institut des Sciences de la Terre d'Orléans" (ISTO). The measurements were done with an IR-MS Delta V Advantage coupled with a LC-Isolink interface (both Thermo Scientific) and a HPLC system serving as a pump for the carrier flow $(100 \mu \mathrm{L}$ of filtered and acidified sample water were directly injected in bulk mode and monitored in continuous flow) (Albéric et al., 2010). The standards used for the $\delta^{13} \mathrm{C}_{\mathrm{DOC}}$ measurements were the internal standard from ISTO $\left(\mathrm{NaHCO}_{3},-4.3 \%\right)$, the benzoic acid ( $-25.7 \%$ ), the USGS-40 (L-glutamic acid, $-26.389 \%$ ), the IAEA-C8 (oxalic acid, $-18.3 \%$ ) and the IAEA-C6 (sucrose, $-10.8 \%$ ). The precision of the isotopic measurements was $0.3 \%$. The $\delta^{13} \mathrm{C}_{\text {DIC }}$ was measured at the Stable Isotopes Laboratory of IPGP. The measurements were done with a GC/IR-MS (Analytical Precision) (Assayag et al., 2006). The standards use for the $\delta^{13} \mathrm{C}_{\text {DIC }}$ measurements were the Rennes II $\left(\mathrm{CaCO}_{3}\right.$ powder, $-9.766 \%)$, the Merck $\left(\mathrm{CaCO}_{3}\right.$ powder, $\left.-8.65 \%\right)$, the Across $\left(\mathrm{CaCO}_{3}\right.$ powder, $\left.0.264 \%\right)$ and the $\mathrm{NaHCO}_{3}(-4.4 \%$ ). The precision of the isotopic measurements was $0.5 \%$.

Spectrophotometric analyses of samples were conducted with a dual beam Evolution 600 UV-Vis - Thermo Scientific spectrophotometer. An aliquot of acidified water sample taken for the DOC measurements was used for the spectroscopic characterization of the dissolved organic matter. Samples were placed in a $1 \mathrm{~cm}$ quartz window cuvette and scanned from 200 to $600 \mathrm{~nm}$, with $1 \mathrm{~nm}$ resolution (Chin et al., 1994).

\section{Results}

\subsection{Major elements}

$\mathrm{pH}$ and concentrations of major elements are presented in Table 2. $\mathrm{pH}$ values are relatively homogeneous, according to the spatial location or hydrological regime of rivers and range from 6.72 to 8.10. Low water level samples have a higher ionic charge (from 1200 to $2300 \mu \mathrm{M})$ than flood level water samples $(<1200 \mu \mathrm{M})$, with ionic charge is the sum of anions $\left(\Sigma^{-}\right)$and cations $\left(\Sigma^{+}\right)$concentrations. The normalized ionic charge balance values (NICB $\left.=\left(\left(\Sigma^{+}-\Sigma^{-}\right) / \Sigma^{+}\right) \square 100\right)$ are generally close to $8 \%$.

The dissolved silica concentrations vary between 110 and $631 \mu \mathrm{M}$. These concentrations are evenly distributed around the Basse-Terre. The dissolved silica concentrations are systematically higher during the low water level than during flood events and are similar to results acquired previously by Rad et al. (2007) in Guadeloupe (from 391 to $654 \mu \mathrm{M}$ ), Rad et al. (2006) in Mont Serrat and by Goldsmith et al. (2010) in Dominica (from 135 to $1048 \mu \mathrm{M}$ during wet season and from 365 to $2110 \mu \mathrm{M}$ during dry season). Moreover, similar silica concentrations for rivers flowing over basaltic rocks in the Deccan Traps, the Mount Cameroun and the Réunion Island were reported by [Louvat and Allègre, 1997], [Dessert et al., 2001] and [Benedetti et al., 2003]. 
The alkalinity is given by the following simplified formula:

$A l k=\left[\mathrm{HCO}_{3}^{-}\right]+2$ ?] $\left[\mathrm{CO}_{3}{ }^{2-}\right]+\left[\mathrm{OH}^{-}\right]-\left[\mathrm{H}^{+}\right]$

Within the $\mathrm{pH}$ range (from 6.72 to 8.10 ) of the rivers, the dominant ion in the alkalinity is $\mathrm{HCO}_{3}{ }^{-}$. Alkalinities range from 148 to $755 \mu \mathrm{M}$, and do not vary spatially. They are higher during low water level than during floods, and they are both similar to results obtained for the Guadeloupe (Rad et al., 2007), Mont Serrat and the Dominica (Rad et al., 2006), and to the lowest values measured in Mount Cameroun rivers (Benedetti et al., 2003) and the Réunion rivers (Louvat and Allègre, 1997).

$\mathrm{HCO}_{3}{ }^{-}$is the dominant anion under low water level conditions, while during floods $\mathrm{Cl}^{-}$ becomes the dominant anion, followed by $\mathrm{SO}_{4}{ }^{2-}$ and $\mathrm{F}^{-}$(Table 2). The high concentrations of $\mathrm{NO}_{3}{ }^{-}$for Deshaies (23rd September 2007) and Beaugendre (10th September 2007) are likely due to the lixiviation of soil horizons enriched in nitrates. The $\mathrm{Cl}^{-}$concentrations increase from the southern part to the northern part of the island, and are the highest during low water level.

Among major cations, $\mathrm{Na}^{+}$is dominant (from 140 to $450 \mu \mathrm{M}$ ). $\mathrm{Ca}^{2+}$ and $\mathrm{Mg}^{2+}$ concentrations vary between 55 and $290 \mu \mathrm{M}$ and 19 and $172 \mu \mathrm{M}$, respectively. $\mathrm{K}^{+}$concentrations range from 11.8 to $31.2 \mu \mathrm{M}$. The cations concentrations distribution is the same around the Basse-Terre. These concentrations are smaller during flood level than during low water level. These cations are in the range of those measured by [Rad et al., 2007] and [Goldsmith et al., 2010]. Cationic concentrations are systematically smaller than the ones reported for rivers flowing through basaltic rocks of the Réunion Island, the Deccan Trap or the Mount Cameroun ( [Louvat and Allègre, 1997], [Dessert et al., 2001] and [Benedetti et al., 2003]).

\subsection{Dissolved inorganic carbon}

Calculated concentrations of DIC are reported in Table 2. The calculations are done with field $\mathrm{pH}$, temperature values and with alkalinity and ionic strength measurements made in the laboratory. The calculated DIC values are obtained with the following simplified formula (neglecting concentrations of $\mathrm{OH}^{-}$and $\mathrm{H}^{+}$):

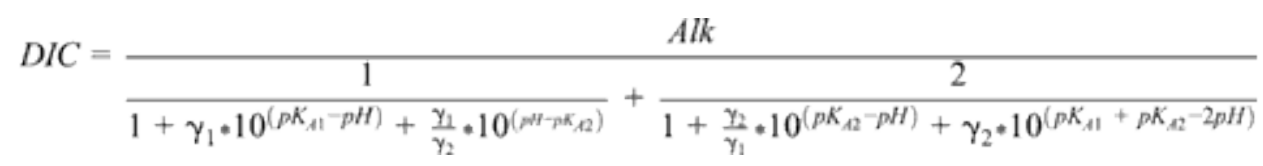

with Alk = alkalinity, $\nu_{1}$ and $\gamma_{2}=$ activity coefficients of ions mono-charged or bi-charged respectively, $\mathrm{K}_{\mathrm{A} 1}$ and $\mathrm{K}_{\mathrm{A} 2}$ corresponding to the first and second acidity constants of carbonate system, respectively.

DIC values range from 149 to $850 \mu \mathrm{M}$ of $\mathrm{C}$. The distribution of DIC concentrations is homogeneous around the Basse-Terre. DIC concentrations are systematically the highest during low water level, and are similar to results obtained by Rivé (2008) in rivers of Martinique (from 319 to $978 \mu \mathrm{M}$ ), of Guadeloupe (from 292 to $817 \mu \mathrm{M}$ ) and of Dominica (from 235 to $962 \mu \mathrm{M}$ ) islands. The calculated partial pressures of the $\mathrm{CO}_{2}\left(\mathrm{pCO}_{2}\right)$ vary between 32 and $299 \mathrm{~Pa}$. The $\mathrm{pCO}_{2}$ variations are not related with hydrological regimes changes since for the northern rivers the values are the highest during low water level and for eastern rivers the values are the highest during floods. Guadeloupean rivers are oversaturated with respect to the atmospheric $\mathrm{CO}_{2}$ according to NOAA (i.e. $39 \mathrm{~Pa}$ from 2006 to 2009, 
www.noaa.gov). These results are similar to values obtained for rivers of Hawaii (Paquay et al., 2007) or for the Xijiang River characterized by a subtropical monsoon (Yao et al., 2007).

The isotopic compositions $\left(\delta^{13} \mathrm{C}_{\mathrm{DIC}}\right)$ are obtained for the same rivers but sampled at different dates. $\delta^{13} \mathrm{C}_{\text {DIC }}$ ranges from -16.7 to $-6.8 \%$, with an average value of $-11.4 \%$ ( $\left.n=20\right)$ during low water level and from -24.4 to $-9.6 \%$, with an average value of $-15.8 \%(n=16)$ during floods. $\delta^{13} \mathrm{C}_{\mathrm{DIC}}$ values are similar to the one measured previously for the Guadeloupean rivers (i.e. from -17.2 to $-7.3 \%$ ) by Rivé (2008).

\subsection{Dissolved organic carbon}

DOC concentrations fall into the range 43 to $601 \mu \mathrm{M}$ of $\mathrm{C}$. DOC concentrations are higher in the northern part than in the rest of the island. DOC concentrations are systemically the highest during flood levels. They are similar to the one measured for small mountainous rivers of New Zealand (from 8 to $630 \mu \mathrm{M}$, mean $30 \mu \mathrm{M}$ for the Southern Alps and $200 \mu \mathrm{M}$ for the southwestern North Island; Carey et al., 2005) and for tropical mountainous rivers of Puerto Rico (mean from 110 to $180 \mu \mathrm{M}$; Mcdowell and Asbury, 1994).

The isotopic compositions of DOC $\left(\delta^{13} \mathrm{C}_{\mathrm{DOC}}\right)$ in studied rivers are given in Table 2 . The $\delta^{13} \mathrm{C}_{\mathrm{DOC}}$ ranges from -32.8 to $-26.2 \%$. The distribution of the isotopic composition of DOC is the same around the Basse-Terre. The $\delta^{13} \mathrm{C}_{\mathrm{DOC}}$ values are systematically more negative during low water level than during floods (Table 2). These values are characteristic of organic matter derived from Guadeloupean tropical forest C3 type plants (Deines, 1980).

The parameters like aromaticity, weight $(\mathrm{Mw})$ and number $(\mathrm{Mn})$ average molecular weight and number and hydrophobic fraction can be estimated from the UV-Vis absorbance data, with empirical relationships (Chin et al., 1994). Different wavelengths are selected $(254,270$, and 280) to underline the markers of organic matter. The absorbance at these different wavelengths defined as SUVA (Specific UV absorbance) is calculated with the following formula:

$$
\begin{gathered}
\text { SUVA }_{X X X}=\frac{\text { Absorbance at wavelength } X X X\left(\mathrm{~cm}^{-1}\right)}{\text { DOC concentration }\left(m g L^{-1}\right)} \\
\left(X X X \text { corresponding to one of the following } \text { SUVA }_{254} ;\right. \\
\text { SUVA } \left._{270} \text { or } \text { SUVA }_{280}\right) .
\end{gathered}
$$

The percentage of aromatic dissolved organic carbon or "aromaticity" is calculated by averaging the values obtained with the relationships proposed by [Chin et al., 1994], [Peuravuori and Pihlaja, 1997] and [Weishaar et al., 2003]. The percentage of aromatic carbon varies between 31 and $75 \%$ (Table 2). The weight averaged molecular weights (Mw) are calculated by averaging the data obtained with the relationships of [Chin et al., 1994] and [Zhou et al., 2001]. The Mw varies between 2521 and 5165 D. The values of the aromaticity and $\mathrm{Mw}$ are relatively similar for all rivers, but these values are higher during low water level than during floods. The percentages of aromatic carbon are similar to results acquired in water samples collected in 16 states of the USA by NASQAN sampling program (Weishaar et al., 2003) and in natural humic-water samples collected from two lakes in 
Finland (Peuravuori and Pihlaja, 1997) using an identical spectroscopic approach. The weight averaged molecular weight values are similar to results obtained for colloidal organic matter from rivers of Amazonian basin (between 1126 and 2230 D, Alasonati et al., 2010). The polydispersity index calculated with ratio $\mathrm{Mw}$ on $\mathrm{Mn}$ was close to $2.0 \pm 0.1$ for all samples, and is identically for all rivers and during low water level and floods.

\section{Discussion}

The following discussion supported by the results presented above will first focus on the role of parameters included in models of watersheds weathering such as average slopes, precipitation and age of the geological substratum on the fluxes of both inorganic and organic carbon. Then the discussion will address the question of the sources of the dissolved organic carbon and their characteristics. Finally, we calculate average annual yields of both organic and inorganic carbon taking into account the spatial and temporal variability on the various studied watersheds the results of these calculations will emphasize the role of islands such as Guadeloupe in the overall global carbon budget.

\subsection{Chemical composition}

Considering our studied area, the global chemical composition of river waters mainly derives from water-rock interaction processes, soil lixiviation and atmospheric inputs. Indeed, samples have been collected upstream of agricultural areas and away from the active volcanic area (South) strongly impacted by hydrothermal springs.

The atmospheric inputs to the rivers can be classically estimated by looking at chlorine concentrations, which are mainly derived from rainwater in areas non-impacted by anthropogenic and geothermal inputs. We have plotted in Fig. 3 sodium concentrations as a function of chlorine concentrations for the different rivers. The $\mathrm{Na} / \mathrm{Cl}$ ratios of rivers are systematically higher than those of rainwater, symbolized by the straight line with a slope of $0.8\left(\mathrm{Na} / \mathrm{Cl}_{\text {rain }}\right)$, emphasizing the additional sources of sodium coming from water-rock interactions. 


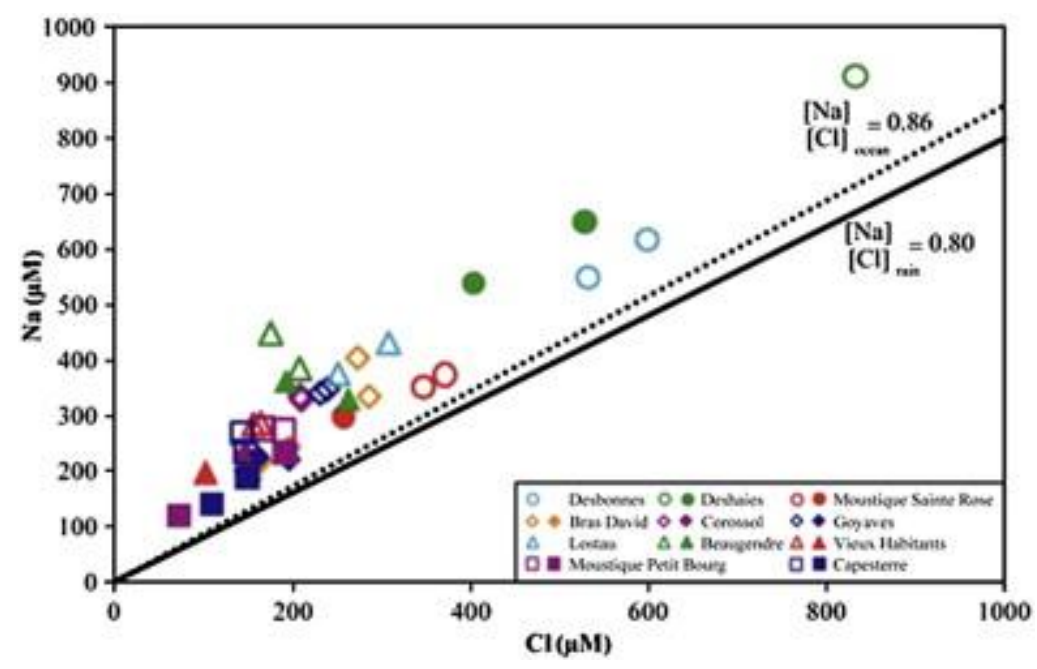

Fig. 3. Sodium concentrations as function of chlorine concentrations expressed in $\mu \mathrm{M}$. The rainfall ratios were obtained from the average concentrations of rainwater sampled in the Southern part of Basse-Terre (Dessert, personal communication). The open and closed symbols correspond to low water level floods, respectively.

Northern rivers present higher concentrations than in the South, due to the highest evapotranspiration factor in the North (Table 1). The sea salt aerosols transported by the Easterly are scavenged by rainwater, which lead to a mean chlorine concentration about $200 \pm 110 \mu \mathrm{M}$ in Guadeloupe (Dessert, personal communication). With evapotranspiration factor $\left(f_{\text {evap }}\right.$ ) varying between 1 and 4 (Table 1 ), we estimate that the concentration of cyclic chlorine in rivers varies between $200 \mu \mathrm{M}$ in the southern part and $800 \mu \mathrm{M}$ for the Deshaies River in the northern part of the island $\left([\mathrm{Cl}]_{\text {river }}=[\mathrm{Cl}]_{\text {rain }}\right.$ ? $\left.\mathrm{f}_{\text {evap }}\right)$. We can thus assume that all the chlorine content has mainly an atmospheric origin. The content of other solutes having an atmospheric origin is determined using riverine chlorine concentrations and rainwater molar ratios $(\mathrm{Na} / \mathrm{Cl}=0.8, \mathrm{Mg} / \mathrm{Cl}=0.09, \mathrm{SO} 4 / \mathrm{Cl}=0.08, \mathrm{Ca} / \mathrm{Cl}=0.06, \mathrm{~K} / \mathrm{Cl}=0.03$, Dessert, personal communication).

The remaining solutes are coming from water-rock interaction processes and are used in the determination of the different chemical weathering rates. TDS cat $_{\text {(cationic total dissolved }}$ solid) is the sum of concentrations of major cations ( $\mathrm{Ca}, \mathrm{Mg}, \mathrm{Na}$ and $\mathrm{K}$ ) corrected for atmospheric inputs. $\operatorname{TDS}_{\mathrm{W}}$ (weathering total dissolved solid) values are calculated from the concentrations of the major dissolved elements originating from andesitic weathering $\left(\mathrm{SiO}_{2}\right.$, $\mathrm{Ca}, \mathrm{Mg}, \mathrm{Na}, \mathrm{K}$ and $\mathrm{SO}_{4}$ ) and corrected for atmospheric inputs. $\mathrm{TDS}_{\text {cat }}$ values vary between 3.7 and $21.4 \mathrm{mg} \mathrm{L}^{-1}$ and $\mathrm{TDS}_{\mathrm{w}}$ concentrations range from 11.8 to $57.5 \mathrm{mg} \mathrm{L}^{-1}$ (Table 2). The TDS values are quite uniform from one area to the other and they are systemically lower during floods, because of a partial dilution effect. $\operatorname{TDS}_{\mathrm{cat}}$ and $\mathrm{TDS}_{\mathrm{w}}$ calculated for Guadeloupean Rivers are similar to those reported in Dessert et al. (2003) for Columbia Plateau, Hawaii, Iceland, the Massif Central and Sao Miguel. 
With mean annual runoff, $\mathrm{HCO}_{3}{ }^{-}$(alkalinity), $\mathrm{TDS}_{\mathrm{cat}}$ and $\mathrm{TDS}_{\mathrm{w}}$ (Table 2) of each rivers, we estimate the mean annual regional rates of $\mathrm{CO}_{2}$ consumption, cationic weathering and chemical weathering for each regions of Guadeloupe.

mean regional $\mathrm{CO}_{2}$ consumption $=\left[\mathrm{HCO}_{3}^{-}\right]_{\text {region }} * R_{\text {region }}$

with $\left[\mathrm{HCO}_{3}{ }^{-}\right]_{\text {region }}=$ mean regional concentration in $\mathrm{HCO}_{3}{ }^{-}$and $\mathrm{R}_{\text {region }}=$ mean regional runoff;

mean regional cationic weathering $=T D$ Scat/region $*$ Rregion

with $\mathrm{TDS}_{\text {cat/region }}=$ mean regional TDS and $\mathrm{R}_{\text {region }}=$ mean regional runoff;

mean regional chemical weathering $=T D S w$,region $*$ Rregion

with $\mathrm{TDS}_{\mathrm{w} / \text { region }}=$ mean regional $\mathrm{TDS}_{\mathrm{w}}$ and $\mathrm{R}_{\text {region }}=$ mean regional runoff and the regions correspond to the Northern, the Center, the Western and the Eastern part of the island.

The rates of $\mathrm{CO}_{2}$ consumption, ranging from 0.26 to $1.3610^{6} \mathrm{~mol} \mathrm{~km}^{-2}$ year ${ }^{-1}$ (Table 3), are similar to the data reported in Rad et al. (2006) for Guadeloupe and Martinique Rivers and in Goldsmith et al. (2010) for the Dominica Rivers. But they are different from the one reported for the Réunion Island (1.3-4.4 $10^{6} \mathrm{~mol} \mathrm{~km}^{-2}$ year $^{-1}$; Louvat and Allègre, 1997) under similar climatic conditions. This is most probably due to the different nature of parent rock (andesitic versus basaltic). The cationic weathering and chemical weathering rates, based on a compilation of $\operatorname{TDS}_{\mathrm{cat}}$ and $\operatorname{TDS}_{\mathrm{w}}$, ranged from 7.8 to 34.0 and 38.9 to $114.5 \mathrm{t} \mathrm{km}^{-2}$ year ${ }^{-1}$ respectively (Table 3). These values are similar to those previously established for the andesitic terrains of Guadeloupe and Martinique (100-

$120 \mathrm{t} \mathrm{km}^{-2}$ year ${ }^{-1}$ ) by Rad et al. (2006) and Dominica (6-106 $\mathrm{t} \mathrm{km}^{-2}$ year ${ }^{-1}$ ) by Goldsmith et al. (2010) and for the basaltic terrains under similar climatic conditions of Reunion Island (63-

$170 \mathrm{t} \mathrm{km}^{-2}$ year $^{-1}$ ) by Louvat and Allègre (1997). These rates vary with geographical location around the Basse-Terre. Furthermore, all Guadeloupean watersheds had chemical weathering rates well in excess of the global mean value of $24 \mathrm{t} \mathrm{km}^{-2}$ year $^{-1}$ (Gaillardet et al., 1999b). Such a difference is expected as large river systems integrate weathering processes occurring on different lithology, different runoff as well as temporary carbon storage or $\mathrm{CO}_{2}$ consumption and production in floodplains ( [Walling et al., 2006], [Bonnet et al., 2008], [Engle et al., 2008] and [Guyette et al., 2008]). The northern rivers of the Guadeloupe Island have the smallest rates compared to the rest of the Island. The smaller rates of the northern part could be due to lower annual precipitations combined with less steep slopes, related to oldest bedrocks. The central, western and eastern parts have higher rates than the northern part, reflecting the high rainfall inputs and the effect of the age of the geological substratum (Gislason et al., 1996). Moreover, for the eastern and western part of the island, the lack of soils will promote faster and stronger weathering as $\mathrm{CO}_{2}$ acid interacts directly with fresh or physically eroded parent rocks. 
Table 3. DOC and DIC yields for the two hydrological stages, and mean annual DOC and DIC yields, as well as calculated mean annual rates of $\mathrm{CO} 2$ consumption, cationic weathering and chemical weathering for different part of the Guadeloupe.

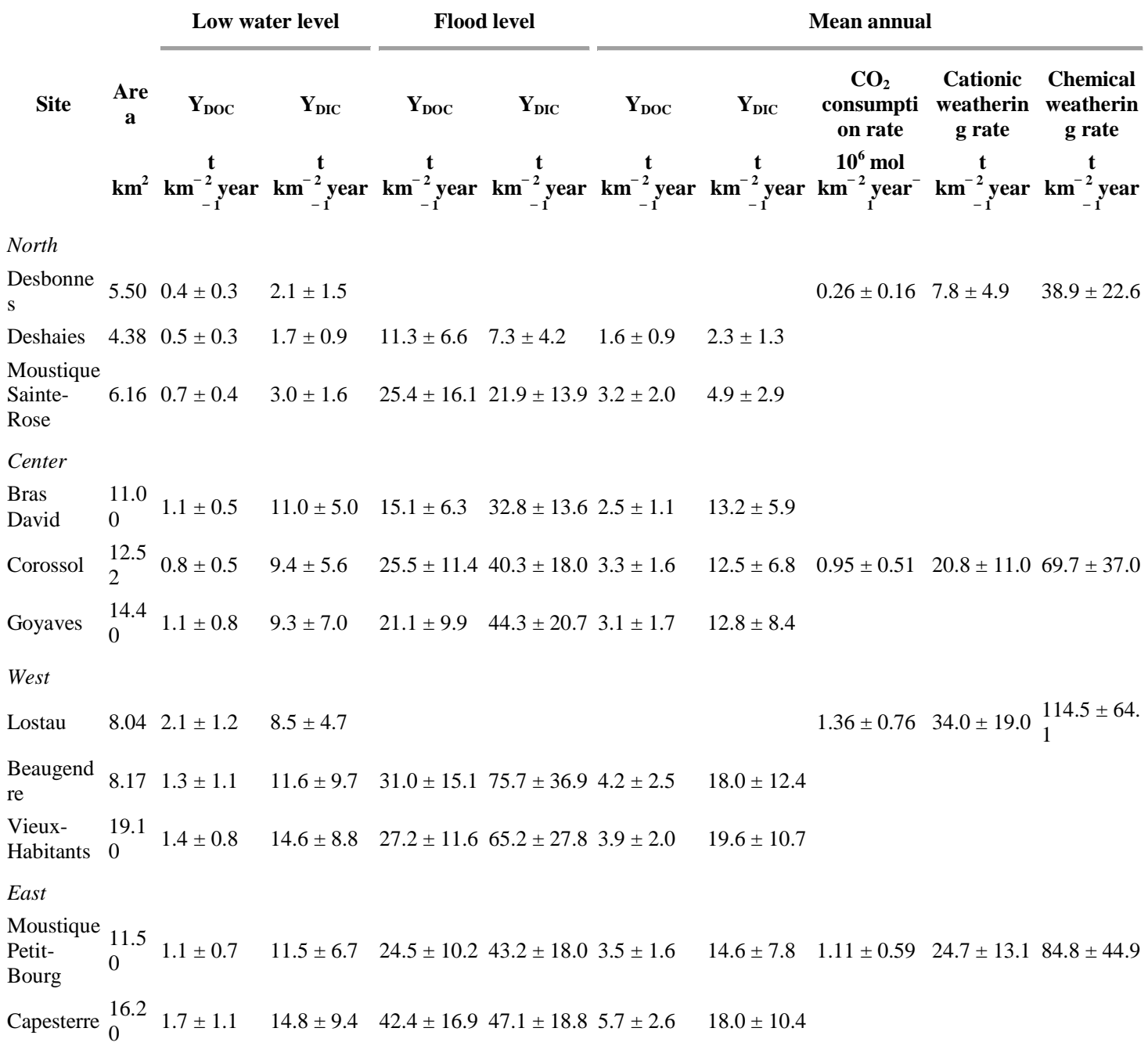

\subsection{The dissolved carbon: spatial and temporal distribution}

DIC concentrations are systematically the highest during low water level (Table 2), and represent more than $75 \%$ of the total dissolved carbon in rivers. During floods, the DIC is partially diluted by rainfall inputs as the lowest calcium and magnesium concentrations cluster in the lower part of Fig. 4. Moreover, the different $\delta^{13} \mathrm{C}_{\text {DIC }}$ values for the two hydrological stages reflect the variable contribution of two end-members. The first one with less negative $\delta^{13} \mathrm{C}_{\text {DIC }}$ corresponds to atmospheric $\mathrm{CO}_{2}$ inputs $(-5$ to $-10 \%$, Trumbore and Druffel, 1995), and the second one corresponds to depleted biogenic $\mathrm{CO}_{2}$ produced during the decomposition of soil organic matter as decomposers used preferentially ${ }^{12} \mathrm{C}$ (Lichtfouse et al., 1995). In Guadeloupean rivers, the major source of the riverine DIC is the $\mathrm{CO}_{2}$ from soils (respiration of plant roots and the decay of organic matter) and atmospheric $\mathrm{CO}_{2}$, both consumed during rock weathering processes (Worall et al., 2005). This hypothesis is supported by the good correlation between divalent cations ( $\mathrm{Ca}$ and $\mathrm{Mg}$ ) concentrations and DIC concentrations given by the Fig. 4 . Elementary ratios of rocks are 0.13 for K/Si, 0.40 for 
$\mathrm{Ca} / \mathrm{Si}, 0.30$ for $\mathrm{Mg} / \mathrm{Si}, 1.33$ for $\mathrm{Ca} / \mathrm{Mg}, 3.00$ for $\mathrm{Ca} / \mathrm{K}$ and 2.30 for $\mathrm{Mg} / \mathrm{K}$ for the $1440 \mathrm{AD}$ eruption of the Soufrière (Poussineau, 2005). In the river waters corrected by the rainfall inputs, the average ratios are $0.03,0.31,0.14,2.23,9.80$ and 4.43 for $\mathrm{K} / \mathrm{Si}, \mathrm{Ca} / \mathrm{Si}, \mathrm{Mg} / \mathrm{Si}$, $\mathrm{Ca} / \mathrm{Mg}, \mathrm{Ca} / \mathrm{K}$ and for $\mathrm{Mg} / \mathrm{K}$, respectively. The differences between water and rock element ratios can be explained by the plant uptake for nutrient elements like potassium (Hinsinger et al., 2001) or by the mixing between of solutions seeping from the weathered soil horizons and the geological substratum. Indeed, the soils have elementary ratios smaller than those of parent rocks (Buss et al., 2010). Moreover, field observations show that the northern and center rivers flow on the soils and not directly on the parent rocks. In addition changes could also be due to the incorporation of Si in secondary minerals such as kaolinite, halloysite, allophane or montmorillonite during incongruent reactions of silicate minerals weathering ( [Colmet-Daage, 1969] and [Colmet-Daage and Bernard, 1979]).

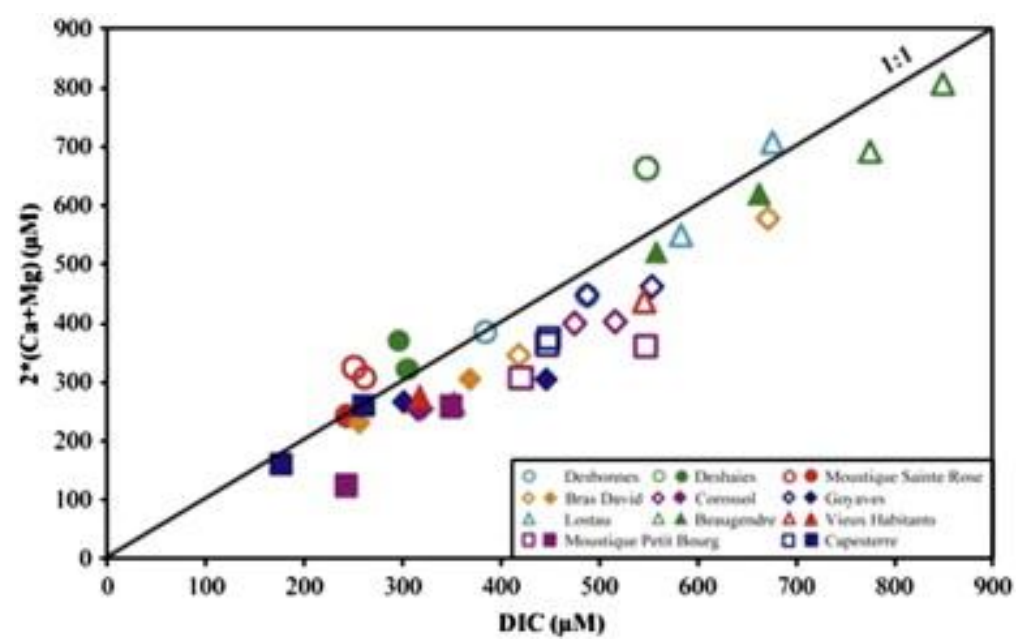

Fig. 4. 2 目 $(\mathrm{Ca}+\mathrm{Mg})$ concentrations as function of DIC concentrations expressed in $\mu \mathrm{M}$. The 1:1 line corresponds to a control of both $\mathrm{Ca}$ and $\mathrm{Mg}$ by weathering processes. DIC is also controlled by the weathering processes of the parent rocks.

Conversely to DIC, DOC concentrations are systematically higher during the flood level than during low water level (Table 2). For the later, the DOC represents from 10 to $30 \%$ of the total carbon, while during floods it accounts for more than $50 \%$ of the total carbon concentration. An input of water enriched with DOC is therefore needed to account for this difference. In Fig. 5, a general negative trend between $\delta^{13} \mathrm{C}_{\mathrm{DOC}}$ versus 1/DOC could correspond to the mixing between two end-members. Data points cluster reasonably along a mixing line with the highest concentrations characterized by less negative $\delta^{13} \mathrm{C}_{\mathrm{DOC}}$ corresponding to flood level and the lowest concentrations associated to the most negative $\delta^{13} \mathrm{C}_{\mathrm{DOC}}$ corresponding to low water level. Variable sources and weathering processes might have obscured that broad relationship between quality and quantity of DOC. One hypothesis would be that during low water level, the rivers could be fed in DOC by autochthonous algal development ( [Kuserk et al., 1984], [Romani et al., 2004], [Sachse et al., 2005] and [Liang et al., 2008]) or by saprolite groundwater ( [Sachse et al., 2005] and [Liang et al., 2008]). Conversely, the high DOC concentrations (116-601 $\mu \mathrm{M}$, Table 2) during floods, could reflect an intense desorption or solubilisation of soil organic matter and its transfer into rivers by surface runoff during these extreme hydrological events. The values of $\delta^{13} \mathrm{C}$ of litter obtained by Rivé (2008), vary between -30.4 and $-27.8 \%$ and are in agreement with those obtained for refractory soil organic carbon (Lichtfouse et al., 1995). In fact, during the decomposition of the $\mathrm{C} 3$ plants $\left(\delta^{13} \mathrm{C}=-20\right.$ to $-35 \%$, Deines, 1980), the 
soil organic matter is enriched by 1.5 to $4.3 \%$ in ${ }^{13} \mathrm{C}$ (Lichtfouse et al., 1995). The $\delta^{13} \mathrm{C}_{\mathrm{DOC}}$ of flood level was in the range of soil litter carbon indicating that the rivers were therefore probably fed by litter layers.

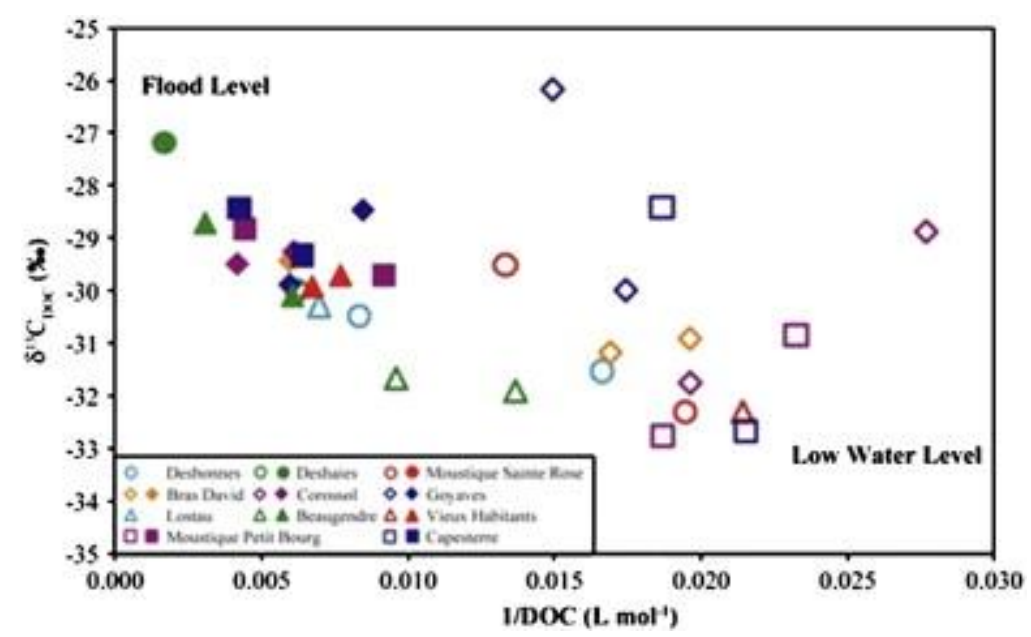

Fig. 5. Isotopic composition of dissolved organic carbon as function of 1/DOC. The general trend suggests a mixing process between two end-members.

Organic matter characteristics (i.e. aromaticity and molecular weight) are reported in Table 2. The DOC aromaticity and the molecular weight estimated for the low water level are higher than the ones corresponding to flood level. These observations are valid for all rivers except the Deshaies river that was not perennial. These parameters combine with isotopic data can help us to decipher between the two potential sources of DOC under low water level conditions (i.e. autochthonous vs saprolite). The trend observed in Fig. 6, between the $\delta^{13} \mathrm{C}_{\mathrm{DOC}}$ and aromaticity has been previously reported (Violleau, 1999). The $\delta^{13} C_{\text {DOC }}$ decreases as DOC aromaticity increases. In the saprolitic horizons, longer water residence time and smaller saprolite-solution ratio prevail. Under such conditions, the more aromatic, more hydrophobic and heavier organic carbon pool could also be desorbed (Muller et al., 2009). In addition at large water fluxes after heavy rainfall events DOM contains more carbohydrates, is less aromatic and more hydrophilic than at small water fluxes (Kaiser and Guggenberger, 2005). Moreover, the autochthonous organic matter production (algal or bacterial production) has, according to Croue (2004), a molecular structure similar to the hydrophilic fraction of natural organic matter. This autochthonous organic matter is generally enriched in ${ }^{13} \mathrm{C}$ and less aromatic. 


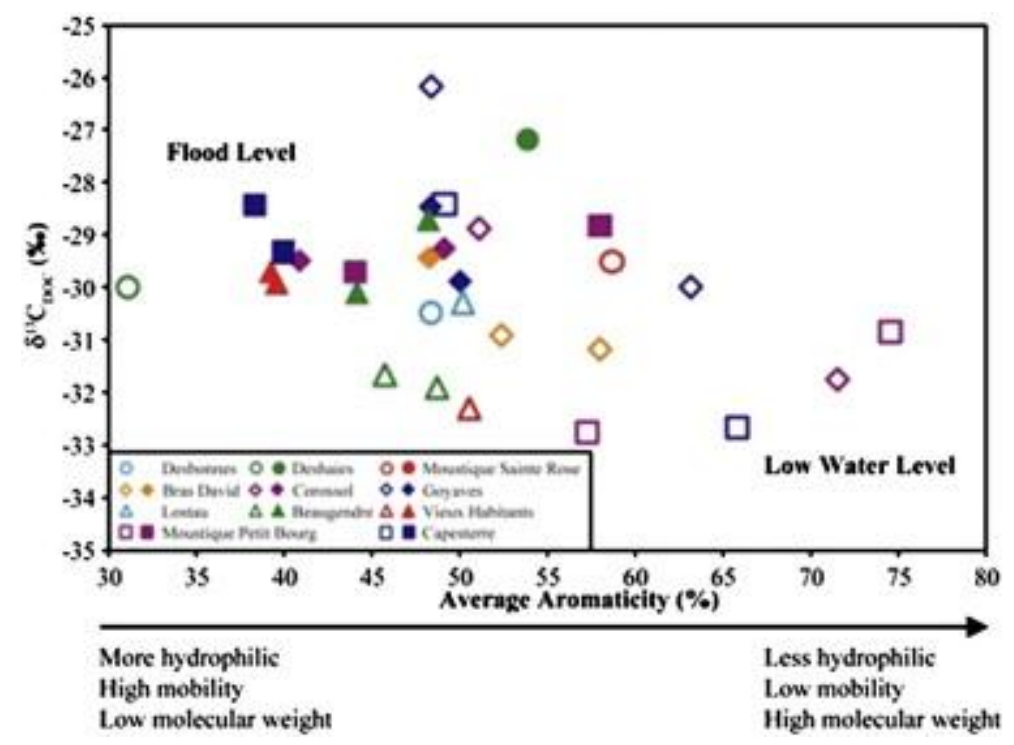

Fig. 6. Isotopic composition of dissolved organic carbon as function of the calculated average $\%$ of aromatic organic carbon. Percentages were obtained by averaging the values calculated with the relationships proposed by [Chin et al., 1994], [Peuravuori and Pihlaja, 1997] and [Weishaar et al., 2003].

In Fig. 7, we summarize the various processes accounting for the observed trends between low water level and floods. During low water level, the rivers are fed by ground flow from the saprolite. The ground flow is depleted in DOC. The organic matter molecules are larger (Mw), more aromatic, less hydrophilic, 13C depleted and less mobile (Cabaniss et al., 2000). Conversely, during floods, the rivers are fed by surface runoff of solutions enriched with DOC. These DOC molecules are smaller, less aromatic, more hydrophilic, 13C enriched and more mobile (Cabaniss et al., 2000). 


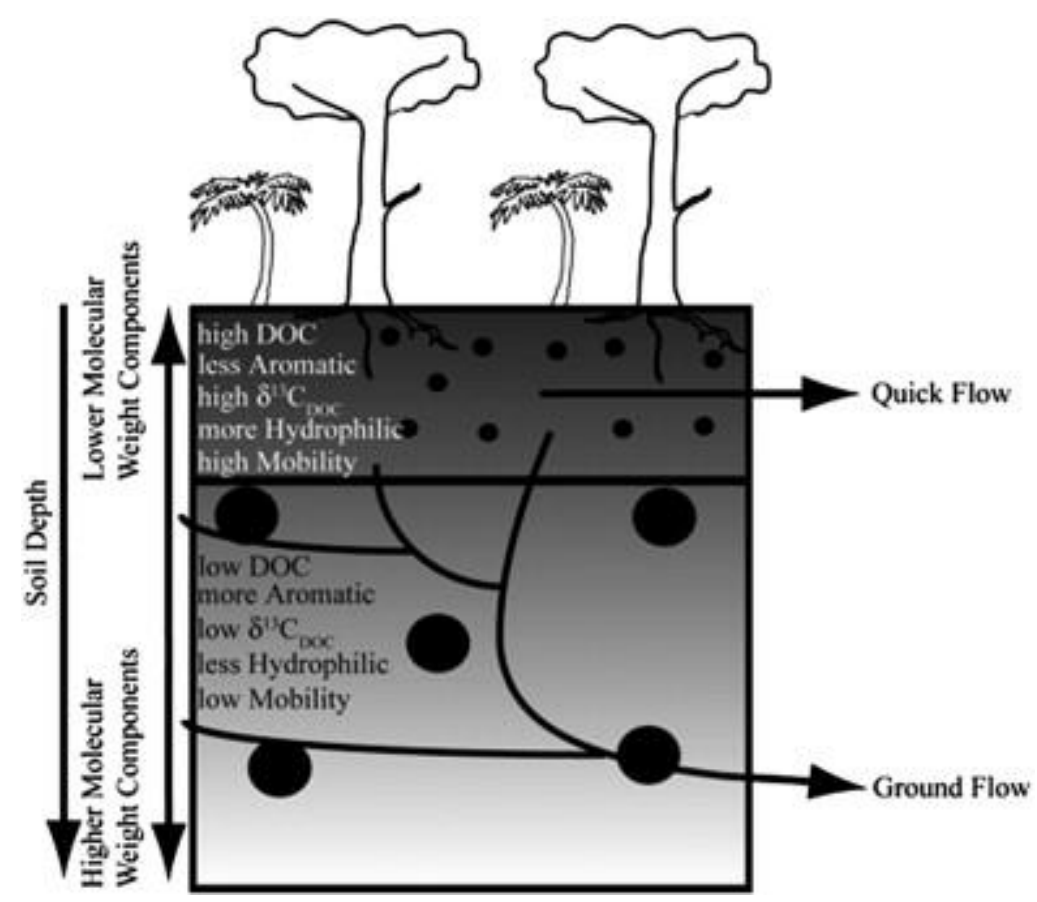

Fig. 7. Summary of the dissolvedorganiccarbon characteristics and potential flowpath in the weathering profile developed on andesitic bedrock of the Basse-Terre Island. During flood events the quick flowpath dominates and the freshly deposited organiccarbon is feeding the rivers. Under low water conditions the ground flowpath is the major source of organic matter for rivers.

The ratio DOC/DIC varies between 0.09 and 0.29 during low water level and between 0.37 and 1.97 during floods (Table 2). The increase of the DOC/DIC ratio for the flood water level is related to the massive input of rainwater that will modify the chemical equilibrium established during the low water level conditions. Both the lower ionic strength of the rainwater and the decrease of soil to pore water ratio will favor the desorption of soil organic matter and its release into the soil solution (Arnarson and Keil, 2000). Under conditions where mineral surfaces become limited and competitive anions are present, such as in surface horizons, newly formed mineral-organic associations are weaker and the $\mathrm{OM}$ is more susceptible to desorption. In contrast, once the availability of mineral surfaces increases in deeper soil horizons, the stabilization of OM upon sorption will increase and only a small portion of dissolvedorganic matter will eventually feed the rivers. This scenario will prevail under low water level conditions and justify the lower DOC/DIC ratios. In Fig. 8, the DOC/DIC values are plotted as function of the percentage of the total watershed area with slopes higher than $49 \%$ (Plaisir et al., 2003). The figure shows that there is a non-linear relationship between the two parameters for both water level conditions. The DOC/DIC ratio decreases as the watershed area is dominated by surfaces with slopes steeper than $49 \%$. The steep slopes will prevent the development of deep soils on the geological substratum and therefore the accumulation of organic matter in the upper horizons that would generate higher DOC in the soil solutions. In addition beyond a threshold value of $10 \%$, the decrease of the DOC/DIC is much smaller. The unknown distribution of organic matter in the soils of the studied watershed might however obscure that broad relationship. 


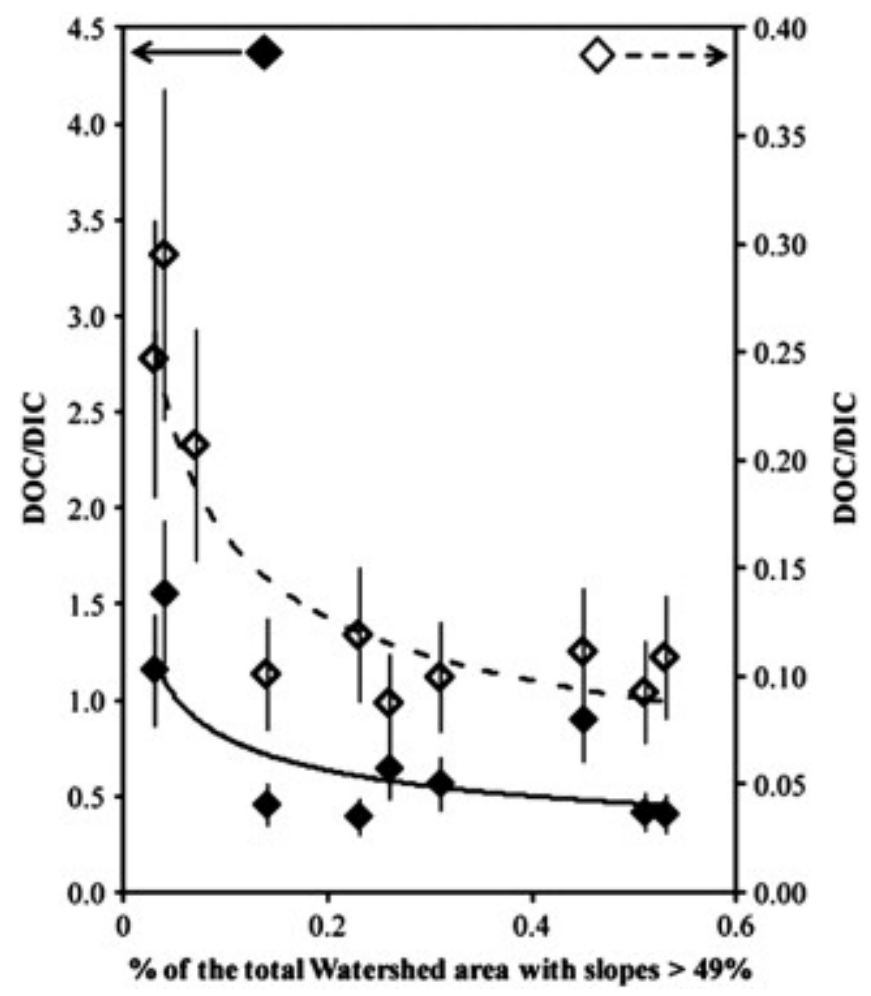

Fig. 8. [DOC]/[DIC] ratio as a function of percentage of the total area of watersheds with slopes $>49 \%$. The open symbols correspond to low water level of rivers and the closed symbols to flood level. Curves are trend lines that correspond to the best fit $(R>0.8)$ between the average slopes of the watersheds. The best fits were obtained for slope above $49 \%$.

\subsection{DOC and DIC yields}

The yields of carbon have been calculated from the mean runoff values and mean carbon concentrations for each river (Table 3). During low water level, the carbonyield varies between 0.4 and $2.1 \mathrm{t} \mathrm{km}^{-2}$ year ${ }^{-1}$ for the DOC and between 1.7 and $14.8 \mathrm{t} \mathrm{km}^{-2}$ year ${ }^{-1}$ for the DIC. The carbonyield during floods varies between 11.3 and $42.4 \mathrm{t} \mathrm{km}^{-2}$ year ${ }^{-1}$ for the DOC and between 7.3 and $75.7 \mathrm{t} \mathrm{km}^{-2}$ year $^{-1}$ for the DIC (Table 3).

The mean annual yields of DOC and DIC were calculated using the following equation:

mean annual yield $=Y_{L W L} * 0.9+Y_{F L} * 0.1$

where $Y_{L W L}$ is the yield during low water level (related to $90 \%$ of the annual discharge; Fig. 2), and $Y_{F L}$ is the yield during floods (related to $10 \%$ of the annual discharge).

The mean annual yields range from 2.5 to $5.7 \mathrm{t} \mathrm{km}^{-2}$ year ${ }^{-1}$ for the DOC and from 4.8 to $19.6 \mathrm{t} \mathrm{km}^{-2}$ year $^{-1}$ for the DIC (Fig. 9). The actual yields of DOC and DIC to the ocean could be different from these calculated yields because our estimation does not take into account extra organic matter inputs from cultivated soils and mangroves located below our sampling points. The erosion of cultivated soils will release organic matter into rivers ( [Bellanger et al., 2004], [Quinton et al., 2006], [Martinez-Mena et al., 2008] and [Gilles et al., 2009]). The real inputs to the ocean are even more difficult to forecast because mangrove can favor the 
accumulation of organic matter from rivers (sink) as well as contribute to the flux of the later by degradation of organic residues issued from the mangrove itself (source) ( [Chen and Twilley, 1999], [Bouillon et al., 2003], [Bouillon et al., 2008], [Chmura et al., 2003] and [Marchand et al., 2006]). The DOC and DIC yields are the most important in the South of the Basse-Terre (Fig. 9). This is likely due to the exposure to rainfalls and steep slopes (related to the younger age of the substratum) which implicate that the erosion of soils is more intense in the southern part than in the northern part of the island. The DOC yield is the most important for the Capesterre River with $5.7 \mathrm{t} \mathrm{km}^{-2}$ year $^{-1}$. The Capesterre watershed is the most exposed to rainfalls and therefore its soils were prone to the erosive power of the extreme meteorological events. The DIC yield is the highest for the Vieux-Habitants River, due to higher weathering rates. The dissolvedcarbonyield is mainly in the form of DIC

(DOC/DIC < 1). However, if we could take into account the particulate organiccarbon then the yield of organiccarbon would be higher than the yield of inorganiccarbon. For instance, the particulate organiccarbon represents about 10-30\% of the total organiccarbon in the Puerto Rico rivers (McDowell and Asbury, 1994). If similar percentages are applied to our samples, the yield of organiccarbon in the Basse-Terre could represent between 18 and $47 \%$ of the total carbonyield. Noteworthy, more than $60 \%$ of the DOC export and $25-45 \%$ of the DIC export occurred during flash floods. 


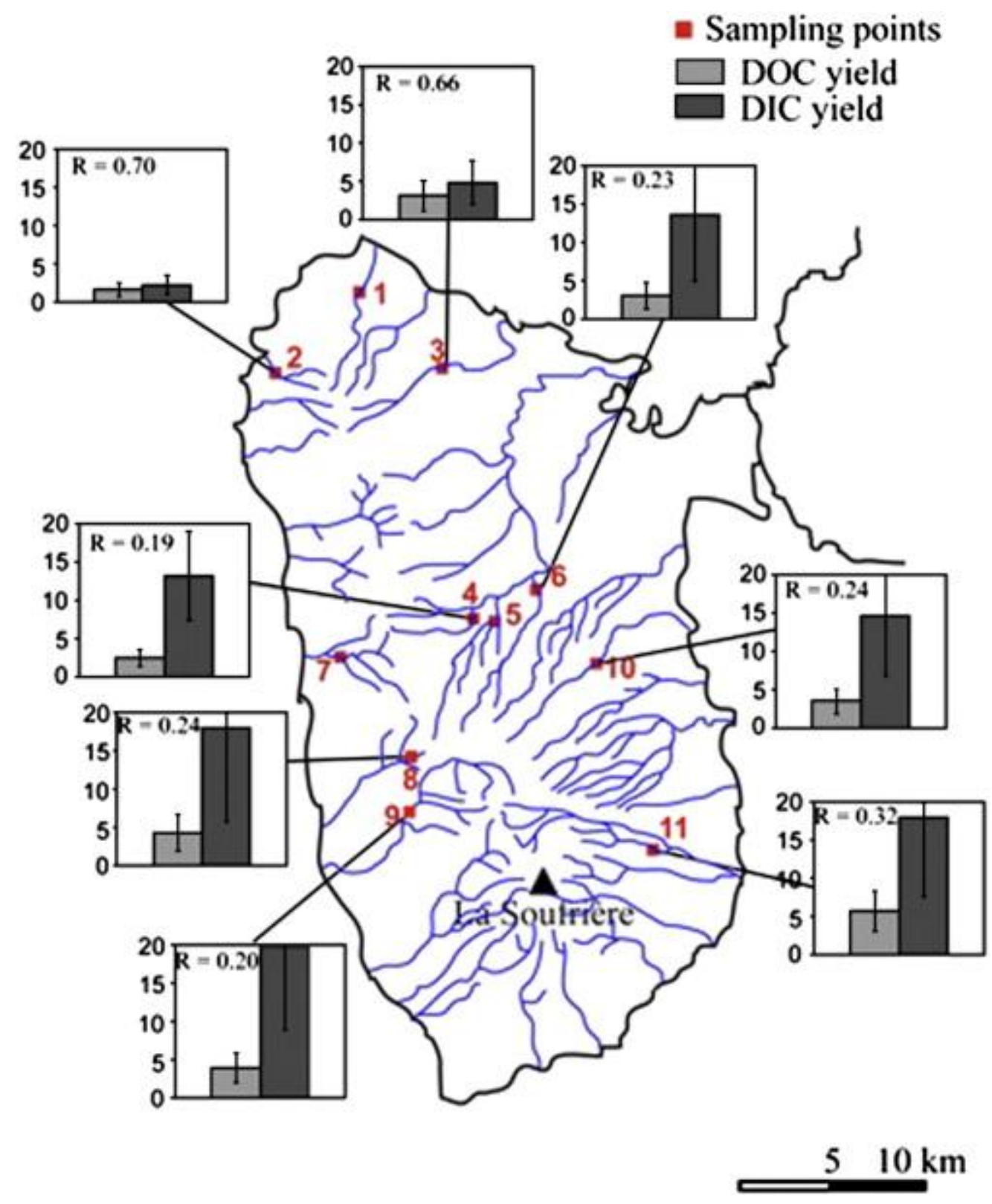

Fig. 9. Average annual DOC and DIC yields ( $\mathrm{km} 2$ year $^{-1}$; Table 3) for 8 watersheds in the Basse-Terre Island. $\mathrm{R}=[\mathrm{DOC}] /[\mathrm{DIC}]$.

The DOC yields are close to the yields calculated at Puerto Rico (Mcdowell and Asbury, 1994) and the yields of New Zealand rivers under temperate climate (Carey et al., 2005). Moreover, the DOC yields are also similar to the yields determined for the large rivers under wet tropical climate like Zaire with $2.5 \mathrm{t} \mathrm{km}^{-2}$ year ${ }^{-1}$ (Nkounkou and Probst, 1987), Parana with $1.4 \mathrm{t} \mathrm{km}^{-2}$ year $^{-1}$ (Depetris and Cascante, 1985), Changjiang with $5.7 \mathrm{t} \mathrm{km}^{-2}$ year $^{-1}$ (Gan et al., 1983), Orinoco with $4.8 \mathrm{t} \mathrm{km}^{-2}$ year $^{-1}$ (Lewis and Saunders, 1989). The DIC yields calculated for the Guadeloupean rivers are more important than the yields determined for the large rivers under wet tropical climate like Zaire with $9.9 \mathrm{t} \mathrm{km}^{-2}$ year ${ }^{-1}$ (Probst, 1992), Parana with $2.0 \mathrm{t} \mathrm{km}^{-2}$ year $^{-1}$ (Cai et al., 2008), Changjiang with $11.2 \mathrm{t} \mathrm{km}^{-2}$ year $^{-1}$ (Cai et al., 2008), Orinoco with $5.5 \mathrm{t} \mathrm{km}^{-2}$ year $^{-1}$ (Cai et al., 2008). The large rivers integrate the yields of several watersheds which present different lithologies and therefore different 
chemical weathering rates and DIC yields. The DIC yields calculated for the Guadeloupean rivers could be the analogue of one kind of sub-catchment in the large rivers systems.

The surface area of volcanic arc islands under wet tropical climate was calculated ( [Louvat, 1997] and [Dessert et al., 2003]) and would represent about $3.29 \cdot 10^{6} \mathrm{~km}^{2}$. We assume that the DOC and DIC yields of Guadeloupean Rivers (1.6-5.7 $\mathrm{t} \mathrm{km}^{-2}$ year $^{-1}$ and 2.3$19.6 \mathrm{t} \mathrm{km}^{-2}$ year $^{-1}$, respectively; Table 3) are representative of DOC and DIC yields export for volcanic arc islands and we estimate therefore the annual DOC and DIC exports of these islands. The DOC and DIC exports from volcanic arc islands under wet tropical climate are about $0.005-0.019 \mathrm{Gt} \mathrm{yr}^{-1}$ and $0.008-0.065 \mathrm{Gt} \mathrm{yr}^{-1}$ respectively. The DOC export is similar to the DOC export calculated for large tropical rivers like the Amazon $\left(0.034 \mathrm{Gt} \mathrm{yr}^{-1}\right.$;

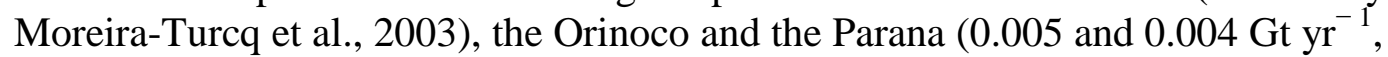
respectively; Ludwig et al., 1996b and references there in) or for the whole African continent $\left(0.025 \mathrm{Gt} \mathrm{yr}^{-1}\right.$; Lal, 2003). The DIC export is close to the DIC export calculated for Africa (0.009 $\mathrm{Gt} \mathrm{yr}^{-1}$; Lal, 2003) or South America (0.059 Gt yr ${ }^{-1}$; Lal, 2003). Therefore, the exports of dissolvedcarbon from small volcanicislands are important and should be included in global carbon budget budgets.

\section{Conclusion}

This study examined the rivers of the volcanicGuadeloupeIsland (FWI) to characterize the yields of dissolvedorganic (DOC) and inorganic (DIC) carbon during weathering in a tropical context. The small rivers present a characteristic hydrological regime where flash floods explain around $10 \%$ of the annual water flux. Dissolvedcarbonyields were constrained by taking into account this hydrological variability with samples collected during both low water and floods for eleven rivers distributed over the island.

Differences in carbon origin during the two hydrological stages were confirmed with DOC and DIC concentrations as well as DOC characteristics and isotopic compositions. During low water level the rivers were mainly fed by soil ground flow while during floods level the rivers were fed by runoff and quick flow.

DOC and DIC yields were estimated with data acquired for both hydrological stages of rivers. Noteworthy, more than $60 \%$ of the DOC export and $25-45 \%$ of the DIC export occurred during flash floods. Neglecting these flash floods leads to an underestimation of the global carbon export from these islands.

A spatial variation of DOC and DIC yields is evidenced around the Basse-Terre Island. Indeed, the DOC and DIC yields are higher in the southern part of the island where the bedrock is younger and the slopes are steeper than in the northern part. Moreover, the southern part receives the maximum of precipitations. 
Therefore, for a carbon mass balance perspective, the exportation of organiccarbon by small tropical rivers can no longer be neglected.

As the particulate organiccarbon represent a non negligible fraction of the total organiccarbonyield in other similar hydrological systems (Puerto Rico), future studies should include particulate organiccarbon characterization from samples taken along the whole year to obtain a full mass balance for the carbon and a better estimation of the organiccarbonyields. In addition organic matter from soils and soil solutions should be characterized to validate the organiccarbon pools proposed in this study to account for the trends between the two hydrological periods.

\section{Acknowledgements}

This work could not be done without logistical support from the OVSG. E. Lajeunesse is acknowledged for his fruitful comments. We thank Guadeloupean DIREN and more precisely M. Pellegrinelli-Verdier for all data on the discharge parameter and his help on the field; the direction (D. Girou) and agents (A. Ferchal, V. Califer) of the National Park of Guadeloupe for all documents and maps on the Guadeloupe generalities; A. Gaillard of LGE for the analysis of dissolved silica; E. Joigneaux of ISTO for her help in the $\delta^{13} \mathrm{C}_{\mathrm{DOC}}$ analysis. This work has been financially supported by the French program funded by the INSU-CNRS (PPF Obsera). $\mathrm{N}^{\circ} 3091$ contribution IPGP. We greatfully acknowledge anonymous reviewers for their helpful and critical comments on the original manuscript.

\section{References}

J.A. Aitkenhead-Peterson, W.H. McDowell, J.C. Neff, E.G.F. Stuart, L.S. Robert Sources, Production, and Regulation of Allochthonous DissolvedOrganic Matter Inputs to Surface Waters Aquatic Ecosystems Academic Press, Burlington (2003)

E. Alasonati, V.I. Slaveykova, H. Gallard, J.-P. Croué, M.F. Benedetti Characterization of the colloidal organic matter from the Amazonian basin by asymmetrical flow field-flow fractionation and size exclusion chromatography Water Research, 44 (2010), pp. 223231

P. Albéric, P. Gautret, D. Juchelka irm-LC/MS: $\delta^{13} \mathrm{C}$ analysis of dissolvedorganic matter in natural waters Thermo Scientific Application, 30198 (2010) 4 pp

P. Amiotte-Suchet, N. Linglois, J. Leveque, A. Andreux $13 \mathrm{C}$ composition of dissolvedorganiccarbon in upland forested catchments of the Morvan Mountains (France): influence of coniferous and deciduous vegetation Journal of Hydrology, 335 (2007), pp. 354-363

T.S. Arnarson, R.G. Keil Mechanisms of pore water organic matter adsorption to montmorillonite Marine Chemistry, 71 (2000), pp. 309-320

N. Assayag, K. Rivé, M. Ader, D. Jézéquel, P. Agrinier Improved method for isotopic and quantitative analysis of dissolvedinorganiccarbon in natural water samples Rapid Communication in Mass Spectrometry, 20 (2006), pp. 2243-2251 
A.K. Aufdenkampe, E. Mayorga, J.I. Hedges, C. Llerena, P.D. Quay, J. Gudeman, A.V. Krusche, J.E. Richey Organic matter in the Peruvian headwaters of the Amazon: compositional evolution from the Andes to the lowland Amazon mainstem Organic Geochemistry, 38 (2007), pp. 337-364

N.H. Batjes Total carbon and nitrogen in the soils of the world European Journal of Soil Science, 47 (1996), pp. 151-163

T.J. Battin, L.A. Kaplan, S. Findlay, C.S. Hopkinson, E. Marti, A.I. Packman, J.D. Newbold, F. Sabater Biophysical controls on organiccarbonfluxes in fluvial networks Nature Geoscience, 1 (2008), pp. 95-100

B. Bellanger, S. Huon, F. Velasquez, V. Valles, C. Girardin, A. Mariotti Monitoring soil organiccarbon erosion with delta $\mathrm{C}^{-1} 3$ and delta $\mathrm{N}^{-1} 5$ on experimental field plots in the Venezuelan Andes Catena, 58 (2004), pp. 125-150

M.F. Benedetti, O. Menard, Y. Noack, A. Carvalho, D. Nahon Water rock interactions in tropical catchments: field rates of weathering and biomass impact Chemical Geology, 118 (1994), pp. 203-220

M.F. Benedetti, A. Dia, J. Riotte, F. Chabaux, M. Gérard, J. Boulègue, B. Fritz, C. Chauvel, M. Bulourde, B. Déruelle, P. Ildefonse Chemical weathering of basaltic lava flows undergoing extreme climatic conditions: the water geochemistry record Chemical Geology, 201 (2003), pp. 1-17

R. Benner What happens to terrestrial organic matter in the ocean? Marine Chemistry, 92 (2004), pp. 307-310

P.C. Bennett, M.E. Melcer, D.I. Siegel, J.P. Hassett The dissolution of quartz in dilute aqueous solutions of organic acids at $25^{\circ} \mathrm{C}$ Geochimica et Cosmochimica Acta, 52 (1988), pp. $1521-1530$

R.A. Berner, A.C. Lasaga, R.M. Garrels The carbonate-silicate geochemical cycle and its effect on atmospheric carbon dioxide over the past 100 millions years American Journal of Science, 284 (1983), pp. 641-683

T.S. Bianchi, J.J. Galler, M.A. Allison Hydrodynamic sorting and transport of terrestrially derived organiccarbon in sediments of the Mississippi and Atchafalaya Rivers Estuarine, Coastal and Shelf Science, 73 (2007), pp. 211-222

M.P. Bonnet, G. Barroux, J.M. Martinez, F. Seyler, P. Moreira-Turcq, G. Cochonneau, J.M. Melack, G. Boaventura, L. Maurice-Bourgoin, J.G. Leon, E. Roux, S. Calmant, P. Kosuth, J.L. Guyot, P. Seyler Floodplain hydrology in an Amazon floodplain lake (Lago Grande de Curuai) Journal of Hydrology, 349 (2008), pp. 18-30

G. Boudon, M.P. Semet, P.M. Vincent Magma and hydro-thermally-driven sector collapses: the 3, 100 and 11, 500 B.P. eruptions of la Grande Découverte (la Soufriere) volcano, Guadeloupe, FrenchWestIndies Journal of Volcanology and Geothermal Research, 33 (1987), pp. 317-323 
Boudon G., Dagain J., Semet M.P., and Westercamp D., 1988. Le massif volcanique de la Soufrière (département de la Guadeloupe, Petites Antilles). In: BRGM (Ed.), Carte géologique au 1/20.000ème.

G. Boudon, J.-C. Komorowski, B. Villemant, M.P. Semet A new scenario for the last magmatic eruption of La Soufrière de Guadeloupe (Lesser Antilles) in 1530 A.D.: evidence from stratigraphy, radiocarbon dating and magmatic evolution of erupted products Journal of Volcanology and Geothermal Research, 178 (2008), pp. 474-490

S. Bouillon, F. Dahdouh-Guebas, A. Rao, N. Koedam, F. Dehairs Sources of organiccarbon in mangrove sediments: variability and possible ecological implications Hydrobiologia, 495 (2003), pp. 33-39

S. Bouillon, R.M. Connolly, S.Y. Lee Organic matter exchange and cycling in mangrove ecosystems: recent insights from stable isotope studies Journal of Sea Research, 59 (2008), pp. 44-58

D.J. Burdige Burial of terrestrial organic matter in marine sediments: a re-assessment Global Biogeochemical Cycles, 19 (4) (2005) http://dx.doi.org/10.1029/2004GB002368

H.L. Buss, A.F. White, C. Dessert, J. Gaillardet, A.E. Blum, P.B. Sak Depth profiles in a tropicalvolcanic critical zone observatory: Basse-Terre, Guadeloupe I.S. Torres-Alvarado, P. Birkkle (Eds.), Proc. of the 13th Intl. Symp. on Water-Rock Interaction (2010)

S.E. Cabaniss, Q.H. Zhou, P.A. Maurice, Y.P. Chin, G.R. Aiken A log-normal distribution model for the molecular weight of aquatic fulvic acids Environmental Science and Technology, 34 (2000), pp. 1103-1109

W.-J. Cai, X. Guo, C.-T.A. Chen, M. Dai, L. Zhang, W. Zhai, S.E. Lohrenz, K. Yin, P.J. Harrison, $\mathrm{Y}$. Wang A comparative overview of weathering intensity and $\mathrm{HCO}_{3}{ }^{-}$flux in the world's major rivers with emphasis on the Changjiang, Huanghe, Zhujiang (Pearl) and Mississippi Rivers Continental Shelf Research, 28 (2008), pp. 1538-1549

A.E. Carey, C.B. Gardner, S.T. Goldshmith, W.B. Lyons, D.M. Hicks Organiccarbonyields from small, mountainous rivers, New Zealand Geophysical Research Letters, 32 (2005), pp. L15404.1-L15404.5

R.H. Chen, R.R. Twilley A simulation model of organic matter and nutrient accumulation in mangrove wetland soils Biogeochemistry, 44 (1999), pp. 93-118

Y.-P. Chin, G. Alken, E. O'Loughlin Molecular weight, polydispersity, and spectroscopic properties of aquatic humic substances Environmental Science and Technology, 28 (1994), pp. 1853-1858

G.L. Chmura, S.C. Anisfeld, D.R. Cahoon, J.C. Lynch Global carbon sequestration in tidal, saline wetland soils Global Biogeochemical Cycles, 17 (2003), pp. 22.1-22.12

F. Colmet-Daage Cartes des sols des Antilles: Guadeloupe volcanique et Martinique au 1/20 000ORSTOM Antilles (1969) 
F. Colmet-Daage, Z. Bernard Contribution à l'Atlas des départements d'Outre-mer: Guadeloupe. Carte des sols de la Guadeloupe, Grande-Terre, Marie-GalanteORSTOM Antilles (1979)

J.-P. Croue Isolation of humic and non-humic NOM fractions: structural characterization Environmental Monitoring and Assessessment, 92 (2004), pp. 193-207

J.J.C. Dawson, C. Soulsby, D. Tetzlaff, M. Hrachowitz, S.M. Dunn, I.A. Malcolm Influence of hydrology and seasonality on DOC exports from three constrating upland catchments Biogeochemistry, 90 (2008), pp. 93-113

De Reynal de Saint-Michel, A., 1966. Carte géologique de Basse-Terre et des Saintes département de la Guadeloupe. In: BRGM (Ed.), Carte géologique au 1/50.000ème.

E.T. Degens, V. Ittekkot Particulate organiccarbon: an overview ,in: E.T. Degens, S. Kempe, R. Herrera (Eds.), Pt. 3. Mitt. Geol.-Paläont. Inst. Univ. Hamburg, SCOPE/UNEP Sonderbd, Transport of Carbon and Minerals in Major World Rivers, 58 (1985), pp. 7-27

P. Deines The carbon isotope composition of reduced organiccarbon P. Fritz, J.C. Fontes (Eds.), Handbook of Environmental isotope Geochemistry, Elsevier, Amsterdam (1980)

C. DeMets, P.E. Jansma, G.S. Mattioli, T.H. Dixon, F. Farina, R. Bilham, E. Calais, P. Mann GPS geodetic constraints on Caribbean-North America plate motion Geophysical Research Letters, 27 (2000), pp. 437-440

P.J. Depetris, E.A. Cascante Carbon transport in the Parana River, in: E.T. Degens, S. Kempe, S. Herrera (Eds.), Transport of Carbon and Minerals in Major World Rivers, Univ, Hamburg, Hamburg (1985), pp. 299-304

C. Dessert, B. Dupré, L.M. François, J. Schott, J. Gaillardet, G. Chakrapani, S. Bajpai Erosion of Deccan Traps determined by river geochemistry: impact on the global climate and the ${ }^{87} \mathrm{Sr} /{ }^{86} \mathrm{Sr}$ ratio of seawater Earth and Planetary Science Letters, 188 (2001), pp. 459-474

C. Dessert, B. Dupré, J. Gaillardet, L.M. François, C.J. Allègre Basalt weathering laws and the impact of basalt weathering on the global carbon cycle Chemical Geology, 202 (2003), pp. 257-273

T. Dittmar, G. Kattner The biogeochemistry of the river and shelf ecosystem of the Arctic Ocean: a review Marine Chemistry, 83 (2003), pp. 103-120

J.I. Drever The effect of land plants on weathering rates of silicate minerals Geochimica et Cosmochimica Acta, 58 (1994), pp. 2325-2332

J.I. Drever Chapter 1: weathering processes O.M. Saether, P. de Caritat (Eds.), Geochemical Processes, Weathering and Groundwater Recharge in Catchment (1997), pp. 1-20

J.I. Drever, L.L. Stillings The role of organic acids in mineral weathering Colloids and Surfaces A: Physicochemical and Engineering Aspects, 120 (1997), pp. 167-181 
S. Duan, T.S. Bianchi, T.P. Sampere Temporal variability in the composition and abundance of terrestrially-derived dissolvedorganic matter in the lower Mississippi and Pearl Rivers Marine Chemistry, 103 (2007), pp. 172-184

B. Dupré, J. Gaillardet, D. Rousseau, C.J. Allègre Major and trace elements of river borne material: the Congo Basin Geochimica et Cosmochimica Acta, 60 (1996), pp. 1301-1321

D.L. Engle, J.M. Melack, R.D. Doyle, T.R. Fisher High rates of net primary production and turnover of floating grasses on the Amazon floodplain: implications for aquatic respiration and regional CO2 flux Global Change Biology, 14 (2008), pp. 369-381

C. France-Lanord, L.A. Derry $\delta^{13} \mathrm{C}$ of organiccarbon in the Bengal fan - source evolution and transport of $\mathrm{C} 3$ and $\mathrm{C} 4$ plant carbon to marine sediments Geochimica et Cosmochimica Acta, 58 (1994), pp. 4809-4814

J. Gaillardet, B. Dupré, C.J. Allègre Geochemistry of large river suspended sediments: silicate weathering or recycling tracer? Geochimica et Cosmochimica Acta, 63 (1999), pp. 4037-4051

J. Gaillardet, B. Dupré, P. Louvat, C.J. Allègre Global silicate weathering and $\mathrm{CO}_{2}$ consumption rates deduced from the chemistry of the large rivers Chemical Geology, 159 (1999), pp. 3-30

V. Galy, C. France-Lanord, B. Lartiges Loading and fate of particulate organiccarbon from the Himalaya to Ganga-Brahmaputra delta Geochimica et Cosmochimica Acta, 72 (2008), pp. $1767-1787$

W.B. Gan, H.-M. Chen, Y.-F. Han Carbon transport by the Yangtze (at Nanjing) and Huanghe (at Jinan) Rivers E.T. Degens, S. Kempe, H. Soliman (Eds.), Transport of Carbon and Minerals in Major World Rivers, Univ. Hamburg, Hamburg (1983)

A.C. Gebhardt, B. Gaye-Haake, D. Unger, N. Lahajnar, V. Ittekot Recent particulate organiccarbon and total suspended matter fluxes from the $\mathrm{Ob}$ and Yenisei Rivers into the Kara Sea (Siberia) Marine Geology, 207 (2004), pp. 225-245

L. Gilles, N.P. Cogo, C.A. Bissani, T. Bagatini, J.C. Portela Water, Soil, organic matter, and nutrient losses by rainfall erosion from an area of native pasture cropped with corn, influenced by tillage methods and fertilization types Revista Brasileira de Ciência do Solo, 33 (2009), pp. 1427-1440

S.R. Gislason, S. Arnorsson, H. Armannsson Chemical weathering of basalt as deduced from the composition of precipitation, rivers and rocks in SW Iceland American Journal of Science, 296 (1996), pp. 837-907

S.T. Goldsmith, A.E. Carey, W.B. Lyons, S.-J. Kao, T.-Y. Lee, J. Chen Extreme storm events, landscape denudation, and carbon sequestration: Typhoon Mindulle, Choshui River, Taiwan Geology, 36 (6) (2008), pp. 483-486

S.T. Goldsmith, A.E. Carey, B.M. Johnson, S.A. Welch, W.B. Lyons, W.H. McDowell, J.S. Pigott Stream geochemistry, chemical weathering and $\mathrm{CO}_{2}$ consumption potential of 
andesitic terrains, Dominica, Lesser Antilles Geochimica et Cosmochimica Acta, 74 (2010), pp. 85-103

M.A. Goñi, K.C. Ruttenberg, T.I. Eglinton A reassessment of the sources and importance of land-derived organic matter in surface sediments from the Gulf of Mexico Geochimica et Cosmochimica Acta, 62 (1998), pp. 3055-3075

P.J. Gregory, J.S.I. Ingram, B. Campbell, J. Goudriaan, L.A. Hunt, J.J. Landsberg, S. Linder, M. Stafford Smith, R.W. Sutherst, C. Valentin Managed production systems B. Walker, W. Steffen, J. Canadell, J. Ingram (Eds.), The Terrestrial Biosphere and Global Change: Implications for Natural and Managed Ecosystems, Cambridge University Press, Cambridge (1999)

R.P. Guyette, D.C. Dey, M.C. Stambaugh The temporal distribution and carbon storage of large oak wood in streams and floodplain deposits Ecosystems, 11 (2008), pp. 643-653

C.J. Hawkesworth, M. Powell Magma genesis in the lesser Antilles island arc Earth and Planetary Science Letters, 51 (1980), pp. 297-308

J.I. Hedges, R.G. Keil, R. Berner What happens to terrestrial organic matter in the Ocean? Organic Geochemistry, 27 (1997), pp. 195-212

R.G. Hilton, A. Galy, N. Hovius, M.C. Chen, M.J. Horng, H.Y. Chen Tropical-cyclone-driven erosion of the terrestrial biosphere from mountains Nature Geoscience, 1 (2008), pp. 759-762

R.G. Hilton, A. Galy, N. Novius, M.-J. Horng, H. Chen The isotopic composition of particulate organiccarbon in mountain rivers of Taiwan Geochimica et Cosmochimica Acta, 74 (2010), pp. 3164-3181

P. Hinsinger, O. Neto Fernandes Barros, M.F. Benedetti, Y. Noack, G. Callot Plant-induced weathering of a basaltic rock: experimental evidence Geochimica et Cosmochimica Acta, 65 (2001), pp. 137-152

D. Ibiza Enquête aux Antilles : influence de la forme de l'averse sur la forme de la crue. Application: méthode globale de prédétermination des crues rares, méthode analytique Cahiers de I'ORSTOM. Série hydrologique, XXI (1984-1985), pp. 99-131

A.D. Jacobson, J.D. Blum, C.P. Chamberlain, D. Craw, P.O. Koons Climatic and tectonic controls on chemical weathering in the New Zealand Southern Alps Geochimica et Cosmochimica Acta, 67 (2003), pp. 29-46

M.S. Johnson, J. Lehmann, E.G. Couto, J.P. Novaes, S.J. Riha DOC and DIC in flowpaths of Amazonian headwater catchments with hydrologically contrasting soils Biogeochemistry, 81 (2006), pp. 45-57

T.H. Jordan The present-day motion of the Caribbean plate Journal of Geophysical Research, 80 (1975), pp. 4433-4439 
K. Kaiser, G. Guggenberger Storm flow flushing in a structured soil changes the composition of dissolvedorganic matter leached into the subsoil Geoderma, 127 (2005), pp. $177-187$

F.T. Kuserk, L.A. Kaplan, T.L. Bott In situ measures of dissolvedorganiccarbonflux in a rural stream Canadian Journal of Fisheries and Aquatic Sciences, 41 (1984), pp. 964-973

R. Lal Soil erosion and the global carbon budget Environment International, 29 (2003), pp. 437-450

R. Lal Soil carbon sequestration impacts on global climate change and food security Science, 304 (2004), pp. 1623-1627

R. Lal Soil erosion and carbon dynamics Soil Tillage Research, 81 (2005), pp. 137-142

W.M. Lewis, J.F. Saunders Concentration and transport of dissolved and suspended substances in the Orinoco river Biogeochemistry, 7 (1989), pp. 203-240

Y. Liang, H.C. Hong, L.H. Dong, C.Y. Lan, B.P. Han, M.H. Wong Sources and properties of natural organic matter (NOM) in water along the Dongjiang River (the source of Hong Kong's drinking water) and toxicological assay of its chlorination by-products Archives of Environmental Contamination and Toxicology, 54 (2008), pp. 597-605

E. Lichtfouse, S. Dou, C. Girardin, M. Grabbly, J. Balesdent, F. Behar, M. Vandenbroucke Unexpected ${ }^{13} \mathrm{C}$-enrichment of organiccarbon components from wheat crop soils: evidence for the in situ origin of soil organic matter Organic Geochemistry, 23 (1995), pp. 865-868

Louvat P., 1997. Etude géochimique de l'érosion fluviale d'îles volcaniques à l'aide des bilans d'éléments majeurs et traces, PhD Thesis, University of Paris-7.

P. Louvat, C.J. Allègre Present denudation rates at Réunion island determined by river geochemistry: basalt weathering and mass budget between chemical and mechanical erosions Geochimica et Cosmochimica Acta, 61 (1997), pp. 3645-3669

W. Ludwig, P. AmiotteSuchet, J.L. Probst River discharges of carbon to the world's oceans: determining local inputs of alkalinity and of dissolved and particulate organiccarbon Comptes Rendus de I'Académie des Sciences Ser II-A, 323 (1996), pp. 1007-1014

W. Ludwig, J.L. Probst, S. Kempe Predicting the oceanic input of organiccarbon by continental erosion Global Biogeochemical cycles, 10 (1996), pp. 23-41

W.B. Lyons, A.E. Carey, D.M. Hicks, C.A. Nezat Chemical weathering in high-sedimentyielding watersheds, New Zealand Journal of Geophysical Research - Earth Surface, 110 (2005), p. 11

C. Marchand, P. Alberic, E. Lallier-Verges, F. Baltzer Distribution and characteristics of dissolvedorganic matter in mangrove sediment pore waters along the coastline of French Guiana Biogeochemistry, 81 (2006), pp. 59-75 
A. Martinez-Mena, J. Lopez, A. Almagro, C. Boix-Fayos, J. Albaladejo Effect of water erosion and cultivation on the soil carbon stock in a semiarid area of South-East Spain Soil Tillage Research, 99 (2008), pp. 119-129

W.H. McDowell, C.E. Asbury Export of carbon, nitrogen, and major ions from three tropical montane watersheds Limnology Oceanography, 39 (1994), pp. 111-125

J.D. Milliman, R. Meade Worldwide delivery of river sediment to the oceans Journal of Geology, 91 (1983), pp. 1-21

J.D. Milliman, P.M. Syvitski Geomorphic/Tectonic control of sediment discharge to the ocean: the importance of small mountainous rivers Journal of Geology, 100 (1992), pp. $525-544$

R. Millot, J. Gaillardet, B. Dupré, C.J. Allègre Northern latitude chemical weathering rates: clues from the Mackenzie River Basin, Canada Geochimica et Cosmochimica Acta, 67 (7) (2003), pp. 1305-1329

A.J. Mills, R.M. Cowling Below-ground carbon stocks in intact and transformed subtropical thicket landscapes in semi-arid South Africa Journal of Arid Environments, 74 (2010), pp. 93-100

P. Moreira-Turcq, P. Seyler, J.L. Guyot, H. Etcheber Exportation of organiccarbon from the Amazon River and its main tributaries Hydrological Processes, 17 (2003), pp. 13291344

M. Muller, C. Alewell, F. Hagedorn Effective retention of litter-derived dissolvedorganiccarbon in organic layers Soil Biology and Biochemistry, 41 (2009), pp. 1066-1074

R.R. Nkounkou, J.L. Probst Hydrology and geochemistry of the Congo River SystemMitt Geol. - Palaont. Inst. Univ. Hamburg, Hamburg (1987)

P. Oliva, J. Viers, B. Dupré, J.P. Fortuné, F. Martin, J.J. Braun, D. Nahon, H. Robain The effect of organic matter on chemical weathering: study of a small tropicalwatershed: Nsimi-Zoétélé site, Cameroon Geochimica et Cosmochimica Acta, 63 (1999), pp. 40134035

F. Pagney Villes de piémont à risques d'inondations en îles tropicales: exemple des Antilles françaises Revue de Géographie Alpine, 4 (1994), pp. 45-57

F.S. Paquay, F.T. Mackenzie, A.V. Borges Carbon dioxide dynamics in rivers and coastal waters of the "Big Island" of Hawaii, USA, during baseline and heavy rain conditions Aquatic Geochemistry, 13 (2007), pp. 1-18

J. Peuravuori, K. Pihlaja Molecular size distribution and spectroscopic properties of aquatic humic substances Analytica Chimica Acta, 337 (1997), pp. 133-149

E.D. Pittman, M.D. Lewan Organic Acids in Geological ProcessesSpringer Verlag (1994) 
Plaisir J., Démonio W., and Claudin J., 2003. Atlas du Parc national de Guadeloupe. In: Ministère de l'Ecologie et du Dévelloppement Durable (Ed.), pp.68.

Poussineau S., 2005. Dynamique des magmas andésitiques : approche expérimentale et pétrostructurale; application à la Soufrière de Guadeloupe et à la Montagne Pelée, Université d'Orléans, PhD thesis.

J.L. Probst Géochimie et Hydrologie de l'Erosion Continentale : Mécanismes, Bilan Global Actuel et Fluctuations au cours des 500 derniers Ma, Sciences Géologiques Mémoire, 94 (1992) Strasbourg

J.N. Quinton, J.A. Catt, G.A. Wood, J. Steer Soil carbon losses by water erosion: experimentation and modeling at field and national scales in the UK Agriculture Ecosystems \& Environment, 112 (2006), pp. 87-102

S. Rad, P. Louvat, C. Gorge, J. Gaillardet, C.J. Allègre River dissolved and solid loads in the Lesser Antilles: new insight into basalt weathering processes Journal of Geochemical Exploration, 88 (2006), pp. 308-312

S.D. Rad, C.J. Allègre, P. Louvat Hidden erosion on volcanicislands Earth and Planetary Science Letters, 262 (2007), pp. 109-124

P.A. Raymond, J.W. McClelland, R.M. Holmes, A.V. Zhulidov, K. Mull, B.J. Peterson, R.G. Striegl, G.R. Aiken, T.Y. Gurtovaya Flux and age of dissolvedorganiccarbon exported to the Arctic Ocean: a carbon isotopic study of the five largest Arctic rivers Global Biogeochemical Cycles, 21 (2007), p. GB4011 http://dx.doi.org/10.1029/2007GB002934

Rivé K., 2008. Origine du carbone inorganique dissous dans les rivières des zones volcaniques: le point de vue du $\delta^{13} \mathrm{C}$. Cas des Antilles, de la Réunion, de I'Islande et du Massif Central, PhD thesis, Université Paris-Diderot.

C. Roelandt, Y. Goddéris, M.-P. Bonnet, F. Sondag Coupled modeling of biospheric and chemical weathering processes at the continental scale Global Biogeochemical Cycles, 24 (2010) http://dx.doi.org/10.1029/2008GB003420

A.M. Romani, H. Guasch, I. Munoz, J. Ruana, E. Vilalta, T. Schwartz, F. Emtiazi, S. Sabater Biofilm structure and function and possible implications for riverine DOC dynamics Microbial Ecology, 47 (2004), pp. 316-328

A. Rousteau Structures, flores, dynamiques: réponses des forêts pluviales des Petites Antilles aux milieux montagnards J.L. Guillaumet, M. Belin, H. Puig (Eds.), Phytogéographie tropicale: réalités et perspectives, ORSTOM, Paris (1996)

A. Sachse, R. Henrion, J. Gelbrecht, C.E.W. Steinberg Classification of dissolvedorganiccarbon (DOC) in river systems: influence of catchment characteristics and autochthonous processes Organic Geochemistry, 36 (2005), pp. 923-935

A. Samper, X. Quidelleur, P. Lahitte, D. Mollex Timing of effusive volcanism and collapse events within an oceanic arc island: Basse-Terre, Guadeloupe archipelago (Lesser Antilles Arc) Earth and Planetary Science Letters, 258 (2007), pp. 175-191 
W.H. Schlesinger Carbon Balance in Terrestrial Detritus Annual Review of Ecology, Evolution and Systematics, 8 (1977), pp. 51-81

B. Schlunz, R.R. Schneider Transport of terrestrial organiccarbon to the oceans by rivers: re-estimating flux- and burial rates International Journal of Earth Sciences, 88 (4) (2000), pp. 599-606

R.F. Stallard Weathering and erosion in the humid tropics A. Lermann, M. Meybeck (Eds.), Physical and Chemical Weathering in Geochemical Cycles, Kluwer, Dordrecht (1988)

R.F. Stallard, J.M. Edmond Geochemistry of the Amazon 2. The influence of geology and weathering environment on the dissolved load Journal of Geophysical Research, 88 (1983), pp. 9671-9688

Y. Sugimura, Y. Suzuki A high-temperature catalytic oxidation method for the determination of non-volatile dissolvedorganiccarbon in seawater by direct injection of a liquid sample Marine Chemistry, 24 (1988), pp. 105-131

S.E. Trumbore, E.R.M. Druffel Carbon isotopes for characterizing sources and turnover of non-living organic matter R.G. Zepp, C. Sonntag (Eds.), Role of Nonliving Organic Matter in the Earth's Carbon Cycle, New York (1995)

J. Viers, B. Dupré, M. Polvé, J. Schott, J.-L. Dandurand, J.J. Braun Chemical weathering in the drainage of a tropicalwatershed (Nsimi-Zoetele site, Cameroune): comparison between organic-poor and organic-rich waters Chemical Geology, 140 (1997), pp. 181206

J. Viers, P. Oliva, J.L. Dandurand, B. Dupré, J. Gaillardet, D.H. Heinrich, K.T. Karl Chemical Weathering Rates, $\mathrm{CO}_{2}$ Consumption, and Control Parameters Deduced from the Chemical Composition of Rivers, Treatise on Geochemistry Pergamon, Oxford (2007)

Violleau D., 1999. Intérêt du fractionnement et de l'extraction des matières organiques naturelles d'eaux de surface pour l'étude de leurs propriétés structurales et de leur pouvoir complexant vis-à-vis du cuivre, PhD Thesis, Université de Poitiers.

G. Wadge Comparison of volcanic production rates and subduction rates at the Lesser Antilles and Central America Geology, 12 (1984), pp. 555-558

D.E. Walling The sediment delivery problem Journal of Hydrology, 65 (1983), pp. 209-237

D.E. Walling, D. Fang, A.P. Nicholas, R.J. Sweet River flood plains as carbon sinks J.S. Rowan, R.W. Duck, A. Werritty (Eds.), Sediment Dynamics and the Hydromorphology of Fluvial Systems, Int Assoc Hydrological Sciences, Wallingford (2006)

M.J. Waterloo, S.M. Oliveira, D.P. Drucker, A.D. Nobre, L.A. Cuartas, M.G. Hodnett, I. Langedijk, W.W.P. Jans, J. Tomasella, A.C. de Araujo, T.P. Pimentel, J.C.M. Estrada Export of organiccarbon in run-off from an Amazonian rainforest blackwater catchment Hydrological Processes, 20 (2006), pp. 2581-2597 
J.L. Weishaar, G.R. Aiken, B.A. Bergamaschi, M.S. Fram, R. Fujii, K. Mopper Evaluation of specific ultraviolet absorbance as an indicator of the chemical composition and reactivity of dissolvedorganiccarbon Environmental Science and Technology, 37 (2003), pp. 47024708

S.A. Welch, W.J. Ullman The temperature dependence of bytownite feldspar dissolution in neutral aqueous solutions of inorganic and organic ligands at low temperature (5$35^{\circ} \mathrm{C}$ ) Chemical Geology, 167 (2000), pp. 337-354

D. Westercamp Magma generation in the Lesser Antilles: geological constraints Tectonophysics, 149 (1988), pp. 145-163

D. Westercamp, H. Tazieff Guides géologiques: Martinique - Guadeloupe - Saint-Martin - La Désirade Masson (Ed.), Guides Géologiques Régionaux. Paris (1980)

F. Worall, W.T. Swank, T. Burt Fluxes of inorganiccarbon from two forested catchments in the Appalachian Mountains Hydrological Processes, 19 (2005), pp. 3021-3035

G. Yao, Q. Gao, Z. Wang, X. Huang, T. He, Y. Zhang, S. Jiao, J. Ding Dynamics of CO2 partial pressure and $\mathrm{CO} 2$ outgassing in the lower reaches of the Xijiang River, a subtropical monsoon river in China Science of The Total Environment, 376 (2007), pp. 255-266

N. Zahibo, E. Pelinovsky, T. Talipova, A. Rabinovich, A. Kurkin, I. Nikolkina Statistical analysis of cyclone hazard for Guadeloupe Lesser Antilles. Atmospheric Research, 84 (2007), pp. 13-29

Q. Zhou, P.A. Maurice, S.E. Cabaniss Size fractionation upon adsorption of fulvic acid on goethite: equilibrium and kinetic studies Geochimica et Cosmochimica Acta, 65 (2001), pp. $803-812$ 